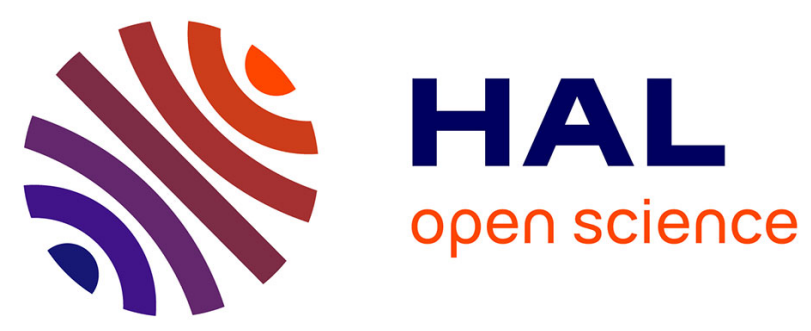

\title{
Theoretical investigation of microalgae culture in the light changing conditions of solar photobioreactor production and comparison with cyanobacteria
}

\author{
Jeremy Pruvost, J.F. Cornet, F. Le Borgne, V. Goetz, J. Legrand
}

\section{To cite this version:}

Jeremy Pruvost, J.F. Cornet, F. Le Borgne, V. Goetz, J. Legrand. Theoretical investigation of microalgae culture in the light changing conditions of solar photobioreactor production and comparison with cyanobacteria. Algal Research - Biomass, Biofuels and Bioproducts, 2015, 10, pp.87-99. 10.1016/j.algal.2015.04.005 . hal-02534118

\author{
HAL Id: hal-02534118 \\ https://hal.science/hal-02534118
}

Submitted on 10 Apr 2020

HAL is a multi-disciplinary open access archive for the deposit and dissemination of scientific research documents, whether they are published or not. The documents may come from teaching and research institutions in France or abroad, or from public or private research centers.
L'archive ouverte pluridisciplinaire HAL, est destinée au dépôt et à la diffusion de documents scientifiques de niveau recherche, publiés ou non, émanant des établissements d'enseignement et de recherche français ou étrangers, des laboratoires publics ou privés. 


\title{
Theoretical investigation of microalgae culture in the light changing conditions of solar photobioreactor production and comparison with cyanobacteria
}

\author{
J. Pruvost ${ }^{\mathrm{a}, *}$, J.F. Cornet ${ }^{\mathrm{b}}$, F. Le Borgne ${ }^{\mathrm{d}}$, V. Goetz $^{\mathrm{c}}$, J. Legrand ${ }^{\mathrm{a}}$ \\ a GEPEA, Université de Nantes, CNRS, UMR6144, bd de l'Université, CRTT-BP 406, 44602 Saint-Nazaire Cedex, France \\ b Clermont Université, ENSCCF, UMR CNRS 6602-Institut Pascal, BP 10448, 63000 Clermont-Ferrand, France \\ c PROMES-CNRS, UPR 8521, Tecnosud, Rambla de la Thermodynamique, 66100 Perpignan, France \\ d AlgoSource Technologies, bd de l'Université, CRTT-BP 406, 44602 Saint-Nazaire Cedex, France
}

\begin{abstract}
A B S T R A C T
Modeling was performed to investigate solar production of the microalga Chlamydomonas reinhardtii in photobioreactors (PBRs). Maximal biomass productivity achievable on Earth was calculated (ideal reactor concept). Effect of PBR location and of given operating conditions was simulated. An ideal productivity (upper limit) in the range of $60 t_{x}$ ha $^{-1}$ year ${ }^{-1}$ was obtained for a fixed horizontal PBR. For a facility sited in France (Nantes), a maximal biomass productivity of around $37 \mathrm{t}_{x} \mathrm{ha}^{-1}$ year $^{-1}$ was predicted.

The comparison against the cyanobacterium Arthrospira platensis highlighted the marked influence of non-ideal light attenuation conditions in the culture volume when growing microalgae. Not only light transmission but also dark volumes were found to negatively impact biomass productivity. Consequently, as biomass growth rate is unable to compensate for rapid changes in sunlight intensity, it proved impossible to maintain optimal light conversion throughout time in outdoor solar conditions. The outcome was a significant decrease in expected productivities, in contrast to cyanobacteria where appropriate optimization resulted in actual productivities approaching the maximal achievable productivities. For microalgae, productivity optimization promoted low light attenuation to safeguard against the marked negative influence of dark volume on microalgae growth. If combined with high PFD, this could impair PBR stability in solar use.
\end{abstract}

\section{Introduction}

Photosynthetic microorganisms such as microalgae and cyanobacteria have emerged as a new high-potential farmable bioresource [1,2]. Their main advantages are solar production with higher surface productivities than plants, and a simultaneous consumption of inorganic carbon which enables carbon-neutral operation. When production is run in closed systems, it offers several additional advantages including an intensified and controlled production with very low environmental impact (no fertilizer is released and water can be re-used). These features, combined with the high biodiversity of microalgae, open up an array of potential feedstock applications as new raw materials for green chemistry or biofuels [3-7].

One of the key aspects in the deployment of microalgal-based industrial applications is the setting of efficient and controlled solar cultivation systems for mass production [2,8-12]. This implies technological development of suitable processes, but also the system-wide optimization of biomass productivity when working in outdoor solar conditions.
The photosynthetic conversion process means that biomass productivity is closely dependent on the light collected. However, unlike other conventional solar technologies such as photovoltaic panels or surfacesupported photocatalysis, light is converted inside a given culture volume, which makes it difficult to predict the resulting productivity as it depends not only on the irradiation conditions but also on the transfer of the collected light flux inside the bulk culture in which light conversion by photosynthetic microorganisms occurs. The prediction of resulting productivity is thus far from trivial. Here, modeling emerges as an especially interesting way forward, as shown by several recent works on solar PBR cultivation [13-18], all of which conclude on the complex relation between irradiation conditions, PBR design, internal light attenuation conditions, and resulting growth. At this juncture, simulation is especially valuable for predicting and optimizing productivities as a function of PBR design, location, and cultivated species.

We recently presented a model able to simulate solar PBR [17-19]. This model was developed for the specific case of light-limited conditions in which light alone limits growth, assuming that all other biological needs (nutrients, dissolved carbon) and operating conditions ( $\mathrm{pH}$, temperature) are controlled at optimal values. It was based on a detailed determination of the irradiance field inside the PBR, taking into account 
specific features of solar use such as (i) the direct/diffuse radiation proportions in sunlight and (ii) time-variation in incident light flux and the corresponding incident angle on the PBR surface. This determination was then coupled with the local kinetics of photosynthetic growth of the cultivated species, paving the way to simulating PBR productivity and its transient response to day-night cycles.

It is well-known that photosynthetic microorganisms absorb light. As a result, photosynthetic growth, and thus process productivity, is deeply affected by light attenuation conditions in the culture volume. In solar conditions, stochastic variation in radiation conditions means that day-long light attenuation conditions are not constant. The process is therefore always running in transient mode, which means efforts to optimize biomass productivity to find a compromise on light attenuation conditions applied in the photobioreactor, for example by controlling biomass concentration value with residence time in the cultivation system. As shown in our previous works $[17,18]$, modeling proved a highly interesting way forward due to its ability to relate variations in radiation conditions, resulting radiative transfers in the culture bulk, and the corresponding photosynthetic conversion and biomass growth kinetics.

In this previous work, modeling was applied to solar cultivation of the cyanobacteria Arthrospira platensis. Cyanobacteria, as prokaryotic cells, have a common electron carrier chain for both photosynthesis and respiration, in contrast to microalgae (eukaryotic cells) which have two different organelles for the two activities (respiration in mitochondria, photosynthesis in chloroplasts). As a result, cyanobacteria display negligible respiration for short residence times in darkness, in contrast to microalgae in which respiration activity is always active [20]. These features greatly influence the resulting productivity in cultivation systems, especially the dark volume created in the culture bulk due to light attenuation. This was clearly shown in Takache et al. [21] in the case of continuous artificial light. With light attenuation conditions presenting dark zones in the culture volume, the biomass productivity of the microalga Chlamydomonas reinhardtii decreased significantly. As any light transmission also resulted in a loss of productivity (as not all available light energy gets absorbed and thus converted), maximal biomass productivity was only found for an optimal biomass concentration value, leading to the so-called "luminostat" regime. Conversely, maximal productivity was achieved for cyanobacteria as soon as full light absorption in the culture volume was obtained with a negligible influence of dark volume in the culture bulk (light transmission resulting in a loss of productivity in any case).

The aim of this work is to investigate consequences of the typical microalgae behavior under changing light attenuation conditions of solar cultivation. Our earlier modeling approach will be extended here to the particular case of solar production of the microalga C. reinhardtii, which we chose based on its status as a model species for eukaryotic photosynthetic microorganisms and its recently published and validated kinetic growth model [21]. As in the case of A. platensis, the model will be used to determine maximal achievable productivity on Earth (mobilizing the concept of "ideal reactor") and to then investigate the influence of geographic location, engineering (i.e., PBR inclination angle) and operating parameters (i.e., residence time) on resulting productivity over a whole-year period. We conclude with a detailed discussion on the light attenuation conditions then encountered in solar operation, emphasizing that the optimization of microalgae cultivation in solar conditions is a more complex challenge than for cyanobacteria.

\section{Methodology}

\subsection{Theoretical considerations}

\subsubsection{Solar photobioreactor modeling}

Recent research has brought a model for simulating PBR operated in solar conditions [17-19]. The main features are given in Appendix A, and the interested reader can refer to the relevant literature. The model relates radiation conditions to biomass production, radiative transfer in the culture volume, and then the resulting photosynthetic growth. By linking the respective kinetics of photosynthetic growth and sunlight variations, the model makes it possible to predict biomass growth represented by time-course of biomass concentration as a function of light collected by the system (Fig. 1). Production can then be determined for a whole-year period, thus yielding data such as biomass productivity.

\subsubsection{Modeling photosynthetic growth for cyanobacteria and microalgae}

Our earlier modeling approach which was restricted to the cyanobacteria case was extended in this work to the solar production of microalgae. Cyanobacteria, as prokaryotic cells, display negligible respiration for short residence times in darkness, in contrast to microalgae in which respiration activity is always active [20]. As a consequence, a dark zone in the culture volume promotes respiration resulting in a loss of biomass productivity. Therefore, achieving the maximum biomass productivity requires in this case the exact condition of complete absorption of the incident light [22], but without a dark zone in the culture volume. This condition is often referred to as luminostat mode.

Maximum biomass productivity can be easily achieved in continuous PBR exposed to artificial constant illumination by setting the biomass concentration corresponding to optimal light attenuation conditions [22]. Under sunlight, the biomass growth rate is not sufficient to compensate for the rapid changes in sunlight intensity. Consequently, light attenuation conditions are never optimal. The purpose of this study was to investigate consequences of such typical behavior of microalgae on solar PBR production.

The consideration of microalgae case implied only to modify the growth kinetic relation in the model (see Appendix A for the remaining parts of the model). To make the kinetic coupling presentation comprehensible two different kinds of metabolism are involved (i.e., cyanobacteria and microalgae), we adopted a unified point of view using a formulation for specific rates of $\mathrm{O}_{2}$ evolution in both cases. The growth kinetic relation giving local photosynthetic specific oxygen evolution rate for the cyanobacterium A. platensis is $[23,24]$ :

$J_{\mathrm{O}_{2}}=\rho \overline{\phi_{\mathrm{O}_{2}}^{\prime}} \mathrm{A} \mathcal{H}\left(G-G_{c}\right)=\rho_{\mathrm{M}} \frac{K}{K+G} \bar{\phi}_{\mathrm{O}_{2}}^{\prime} E_{a} G \mathcal{H}\left(G-G_{c}\right)$

where $\mathcal{H}\left(G-G_{c}\right)$ is the Heaviside function $\left(\mathcal{H}\left(G-G_{c}\right)=0\right.$ if $G<G_{c}$ and $\mathcal{H}$ $\left(G-G_{c}\right)=1$ if $\left.G>G_{c}\right), \rho_{\mathrm{M}}$ is maximum energy yield for photon conversion, $\overline{\phi^{\prime}}$ is the mole $\mathrm{O}_{2}$ quantum yield for the $Z$ scheme of photosynthesis, and $K$ is the half-saturation constant for photosynthesis.

This equation is valid for prokaryotic cells like A. platensis that display negligible short-time respiration in darkness. For microalgae (eukaryotic cells), growth in light would be the result of the evolution in $\mathrm{O}_{2}$ caused by photosynthesis in chloroplasts and its partial degradation by respiration in mitochondria. It is thus necessary to introduce an appropriate kinetic formulation taking into account respiration at light. This was recently proposed for the microalga $C$. reinhardtii in Takache et al. [21], leading to a similar form to Eq. (1):

$$
\begin{aligned}
J_{\mathrm{O}_{2}} & =\left[\rho \bar{\phi}_{\mathrm{O}_{2}}^{\prime} \mathcal{A}-\frac{J_{\mathrm{NADH}_{2}}}{v_{\mathrm{NADH}_{2}-\mathrm{O}_{2}}} \times \frac{K_{r}}{K_{r}+G}\right] \\
& =\left[\rho_{M} \frac{K}{K+G} \bar{\phi}_{\mathrm{O}_{2}}^{\prime} \mathcal{A}-\frac{J_{\mathrm{NADH}_{2}}}{v_{\mathrm{NADH}_{2}-\mathrm{O}_{2}}} \times \frac{K_{r}}{K_{r}+G}\right] .
\end{aligned}
$$

This formulation introduces a specific term relating the negative contribution of respiration activity to overall growth rate. In this term, $J_{\mathrm{NADH}_{2}}$ is the specific rate of cofactor regeneration on the respiratory chain, linked to oxygen consumption by the stoichiometric coefficient $v_{\mathrm{NADH}_{2}-\mathrm{O}_{2}}$ (the stoichiometric coefficient of cofactor regeneration on the respiratory chain), and $\mathrm{K}_{\mathrm{r}}$ is a saturation constant describing the 


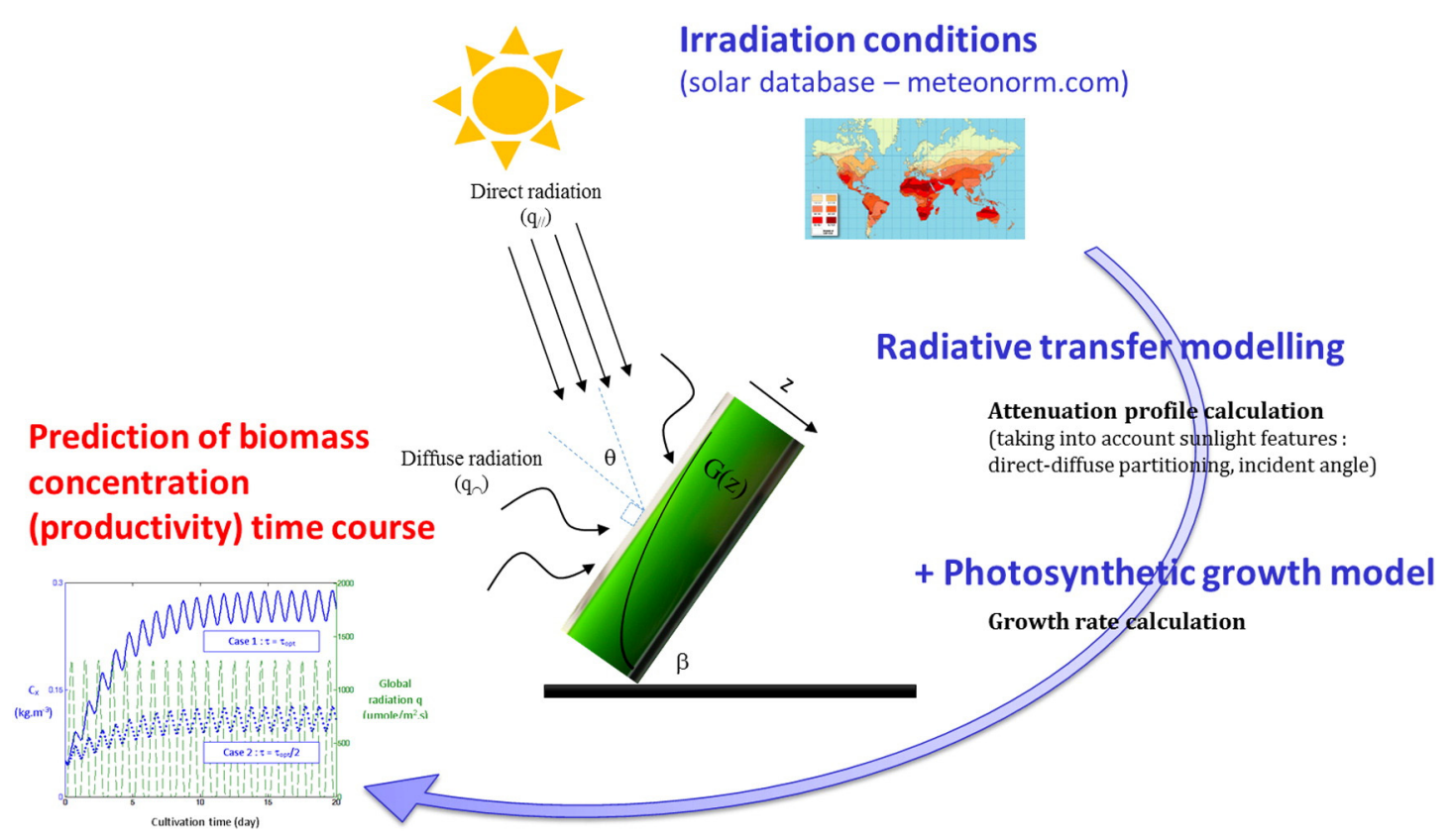

Fig. 1. Overview of the general modeling approach used to simulate solar PBR (for details, see Appendix A).

inhibition of respiration in light. As described in Takache et al. [21], $\mathrm{K}_{\mathrm{r}}$ is entirely determined by the known irradiance of compensation $\left(J_{\mathrm{O} 2}\left(G_{\mathrm{c}}\right)=0\right)$ when the specific respiration rate $J_{\mathrm{NADH}_{2}}$ is known:

$$
\mathrm{K}_{\mathrm{r}}=\frac{G_{c}}{\frac{J_{\mathrm{NADH}_{2}}}{v_{\mathrm{NADH}_{2}-\mathrm{O}_{2}} \rho_{\mathrm{M}} \bar{\phi}_{\mathrm{O}_{2}}^{\prime} E_{a}}\left[\frac{1}{G_{c}}+\frac{1}{K}\right]-1} .
$$

Consequently, $\mathrm{K}_{\mathrm{r}}$ is not an independent parameter of the model.

Finally, in the same manner as for prokaryotic or eukaryotic microorganisms, the mass volumetric biomass growth rate is simply given (from Eqs. (1) or (2)) by the mole-to-mass conversion:

$r_{X}=\frac{J_{\mathrm{O}_{2}} C_{X} M_{X}}{v_{\mathrm{O}_{2}-X}}$

in which $v_{\mathrm{O}_{2}-X}$ takes two different values for nitrate or ammonium as $\mathrm{N}$ sources (see Table 1 ).

Eq. (4) was finally used in the model described in Appendix A to obtain time course of biomass concentration as a function of PBR operating conditions, and then to calculate the corresponding surface biomass productivity $P_{S}\left(\mathrm{~g} \cdot \mathrm{m}^{-2} \cdot\right.$ day $\left.^{-1}\right)$.

For the sake of clarity, all the parameters involved in the coupling kinetic growth models for both $A$. platensis and $C$. reinhardtii are given in Table 1 . This enables to verify the genericity of the proposed approach and its possible extension to other microorganisms.

\subsubsection{Consideration of night period and photoacclimation process}

Eqs. (1) and (2) are valid insofar as the culture is illuminated (i.e., during daytime). At night, long dark periods of several hours trigger a switch to respiratory metabolism with a resulting biomass catabolism $[25,26]$. This can be taken into account by introducing a negative biomass decay rate of production for night periods. For A. platensis and $C$. reinhardtii, values of $\left\langle r_{x}>/ C_{X}=\mu=0.001 \mathrm{~h}^{-1}\right.$ and $0.004 \mathrm{~h}^{-11}$, respectively, were recorded at their optimal growth temperature, i.e., $308 \mathrm{~K}$ for A. platensis and $293 \mathrm{~K}$ for $C$. reinhardtii $[27,28]$. Those constant specific rates were applied during night periods. Note too that both formulations assume standard light-limited photosynthetic growth conditions. Photoinhibition effects are, for example, not considered, although they could arise for intense solar radiation. As a rule, working with complete light extinction in the culture and good mixing will greatly reduce photoinhibition effects [2].

It is also well-known that photosynthetic microorganisms demonstrate photoacclimation, i.e., their pigment content evolves as a function of light conditions $[2,29]$. This affects directly radiative properties of the cells [30,31]. For C. reinhardtii, Takache et al. [21] calculated the evolution in radiative properties of $C$. reinhardtii (here in continuous artificial light) as a result of the decrease in pigment content with increasing PFD. Except when stated otherwise, this relation was introduced in our model, assuming then, in a first instance, a photoacclimation to the monthly average PFD (this assumption is discussed further down).

\subsection{Definition of simulation cases}

\subsubsection{PBR geometry and algae strain}

A rectangular PBR was retained for the simulation with an arbitrary depth $L=0.1 \mathrm{~m}$ (single standing unit with no shadowing). The same approach would be valid for any other PBR depth or PBR geometry such as tubular or cylindrical, with the appropriate radiative transfer model. Note however that when expressed per unit surface area and under light limitation, productivity for a given geometry is independent of PBR depth [32,33].

C. reinhardtii was retained as a model microalgae species as the kinetic equation of its growth is available [21]. As discussed in previous work, maximum productivities in PBRs are also dependent on $\mathrm{N}$ source used to grow the algae $[18,19,34,35]$. As $C$. reinhardtii can grow on ammonium as $\mathrm{N}$-source, choosing this strain enables complementarity with our previous work on A. platensis which grows on nitrate as Nsource.

\subsubsection{PBR implantation and solar radiation conditions}

Two locations were considered, namely Dongola (Sudan, $19.1^{\circ} \mathrm{N}-$ $30.3^{\circ} \mathrm{W}$ ) which represents one of the best locations on Earth in terms of solar irradiation $\left(2476 \mathrm{kWh} \cdot \mathrm{m}^{-2} \cdot\right.$ year $^{-1}$ for a horizontal fixed system, as obtained with METEONORM software), and Nantes (France, $47^{\circ} 12 \mathrm{~N}, 01^{\circ} 33 \mathrm{~W}, 1220 \mathrm{kWh} \cdot \mathrm{m}^{-2} \cdot$ year $^{-1}$ for a horizontal system, which typically gets half of the maximum irradiation in Dongola). Both locations provide very different irradiation conditions in terms of 
energy received, incident angle, and direct-diffuse distribution (see Pruvost et al. [18] for details).

Radiation conditions were obtained using commercial software integrating solar models (METEONORM 6.0 software, www.meteonorm. $\mathrm{com}$ ). The software was used to generate yearly time-course plots of irradiation on the PBR surface (as characterized by its inclination and orientation) for given geographical locations and production periods. Solar data included time variation of direct radiation $q_{/ /}$, diffuse radiation $q_{\cap}$, incident angle $\theta$ and direct normal radiation $q_{\perp}$. All radiation data involving the whole solar spectrum at ground level [0.26-3 $\mu \mathrm{m}]$ were corrected to the photosynthetically active radiation (PAR) of incident light flux densities [0.4-0.7 $\mu \mathrm{m}$ ], which accounts for almost $43 \%$ of the full solar energy spectrum (AM 1.5).

\subsubsection{Ideal surface-lighted $P B R$}

The concept of ideal reactor defines the upper theoretical limit in terms of kinetic performance, as commonly used in chemical engineering [36] and we have already applied it to the case of solar PBR [19]. Exactly the same approach was used here but for the microalga C. reinhardtii instead of the cyanobacteria A. platensis. The ideal PBR was defined as a system located at the equator and at sea level with an ideal atmosphere (no diffuse light, $q_{\cap}=0$ ). This gives the maximum irradiation conditions potentially available at the Earth's surface, i.e., roughly $3500 \mathrm{kWh} \cdot \mathrm{m}^{-2} \cdot$ year $^{-1}$ (total spectrum) or $1500 \mathrm{kWh} \cdot \mathrm{m}^{-2} \cdot \mathrm{year}^{-1}$ (PAR range), which corresponds to a dailyaveraged hemispherical light flux density, or PFD, of $\bar{q}_{\perp} \cong 340 \mathrm{~W} \cdot \mathrm{m}^{-2} \cong$ $1560 \mu$ mole $\cdot \mathrm{m}^{-2} \cdot \mathrm{s}^{-1}$ (expressed here in the PAR range). This value implies normal incidence maintained throughout the day and thus continuous tracking of the sun's path. For a fixed system, variation in the incident angle $\theta$ reduces the amount of light collected by the PBR and also influences radiative transfer conditions in the culture volume, which essentially decreases biomass growth rate. This means we have to factor in the daily variation in incident angle, which will depend on PBR location and tilt angle. For a flat horizontal surface system, this gives $\bar{q}=\bar{q}_{/ /}$ $\cong 250 \mathrm{~W} \cdot \mathrm{m}^{-2} \cong 1150 \mu \mathrm{mole} \cdot \mathrm{m}^{-2} \cdot \mathrm{s}^{-1}(\overline{\cos \theta}$ value of $2 / \pi=0.636)$ corresponding to a received energy of around $2550 \mathrm{kWh} \cdot \mathrm{m}^{-2} \cdot \mathrm{year}^{-1}$ (total spectrum) or $1100 \mathrm{kWh} \cdot \mathrm{m}^{-2} \cdot \mathrm{year}^{-1}$ (PAR range). Because horizontal inclination is the best inclination at the equator location, this corresponds to the ideal conditions of illumination for a fixed PBR.

Once the radiation conditions of the ideal day have been defined, ideal productivity is obtained assuming optimal operating conditions (the best-case scenario, see Appendix A). Note here that the concept of ideal PBR is applied in exactly the same manner for cyanobacteria and microalgae, as optimum conditions entail running in luminostat regime (and thus no dark zone that would otherwise greatly influence microalgae growth, as explained later). As a matter of fact, this only results in a change in the model constants related to the species cultivated and subsequently used to calculate the ideal productivity.

\section{Results and discussion}

\subsection{Areal productivity of an ideal surface-lighted PBR}

The concept of ideal reactor enables us to calculate the maximal productivities achievable on Earth in terms of biomass productivity. Such information is obviously of prime relevance in a mass-scale production setting. For $C$. reinhardtii, areal productivities of $100 \mathrm{t}_{x} \cdot \mathrm{ha}^{-1} \cdot \mathrm{year}^{-1}$ and $60 \mathrm{t}_{\mathrm{x}} \cdot \mathrm{ha}^{-1} \cdot$ year $^{-1}$ were obtained here for sun-tracking and fixedhorizontal ideal PBRs, respectively. The sun-tracking condition logically leads to $40 \%$ higher productivity than the fixed horizontal system and resulting non-normal incidence condition which decreases light received but also increases light attenuation conditions in the culture volume (see Pruvost et al. [18]). Comparing these results against those obtained for the cyanobacteria $A$. platensis, here $C$. reinhardtii demonstrates (around 40\%-50\%) higher productivity. This is mainly explained by the more growth-efficient nitrogen source (ammonium instead of nitrate), dependent on the related $v_{\mathrm{O}_{2}-X}$ value (see Table 1 ), which alone accounts for around 35\% of the higher growth. These productivity values obtained for an ideal PBR are retained here as reference for all further discussion.

In addition to the numerical resolution of Eq. (A6) (time resolution), we also propose a simpler engineering method to assess maximal expected productivity for a given PBR and species, based on an analytical approximation solution which was developed in [19]:

$<s_{X}>_{\max }=\rho_{\mathrm{M}} \frac{\bar{\phi}_{\mathrm{O}_{2}}^{\prime} M_{X}}{v_{\mathrm{O}_{2}-X}} \frac{2 \alpha}{1+\alpha}\left[\frac{\bar{x}_{d} K}{2} \ln \left[1+\frac{2 \bar{q}}{K}\right]+\left(1-\bar{x}_{d}\right) \overline{\cos \theta} K \ln \left[1+\frac{\bar{q}}{K \overline{\cos \theta}}\right]\right]$

where $\bar{q}$ is the mean total incident PFD and $\bar{x}_{d}$ is its mean diffuse fraction at a given location, and $\overline{\cos \theta}$ takes into account the angle $\theta$ between the outward normal of the PBR and the sun's course at a given location (for example, an ideal value of $2 / \pi=0.64$ for a horizontal PBR at the equator).

This equation is restricted to the calculation of maximal productivity (i.e., the equation only holds for the case of optimal operating conditions, Conditions 1 and 2 in Appendix A). It can be applied to either microalgae or cyanobacteria (as both share Condition 1, i.e., luminostat regime). In the present case, using Eq. (5) the horizontal fixed PBR achieved $61 \mathrm{t}_{x} \cdot \mathrm{ha}^{-1} \cdot$ year $^{-1}$ and the sun-tracking system achieved $102 t_{x} \cdot h^{-1} \cdot$ year $^{-1}$. These results are very close to values achieved by full-year dynamic simulation as obtained in the previous section. This again proves the consistency of the proposed formula, as already proved in several cases including solar and artificial light production [18], with systems of various geometries (flat panels, but also cylindrical systems). The deviation, compared to the full simulated values, is negligible. The equations proved to give a good engineering estimation of maximum achievable productivity based on the knowledge of only a handful of kinetic parameters and incident radiation conditions easily available from meteorological databases.

\subsection{Maximal areal productivity of an actual fixed surface-lighted PBR}

Consistent with the definition of an ideal reactor which fixes the upper limit of achievable productivity for a given species, any realworld PBR will have lower performances owing to:

- non-ideal irradiation conditions due to location, meteorological conditions, partial shading by other units or surrounding buildings or trees,

- transient response of the PBR due to biological kinetics, daytime variation in irradiation and night periods,

- poor control of the radiation field leading, for example, to a kinetic regime, and

- engineering (PBR orientation and inclination, dark volumes in the system), technical (optical transmittances, etc.) and/or operating constraints (non-ideal temperature or $\mathrm{pH}$, non-optimized harvesting

Table 1

Summary of the kinetic model parameters.

\begin{tabular}{|c|c|c|c|}
\hline & A. platensis & C. reinhardtii & \\
\hline Parameter & Value & Value & Unit \\
\hline$\rho_{\mathrm{M}}$ & & 0.8 & - \\
\hline$J_{\mathrm{NADH}_{2}}$ & - & 2.3 & $\mathrm{~mol}_{\mathrm{O}_{2}} \mathrm{~kg}_{\mathrm{X}}^{-1} \mathrm{~h}^{-1}$ \\
\hline$v_{\mathrm{O}_{2}-X}$ & 1.44 (nitrate) & 1.08 (ammonium) & - \\
\hline$\overline{\phi^{\prime}}$ & & $1.1 \times 10^{-7}$ & $\mathrm{~mol}_{\mathrm{O} 2} \cdot \mu \mathrm{mol}_{h \nu}{ }^{-1}$ \\
\hline$M_{X}$ & & 0.024 & $\mathrm{~kg}_{X} \mathrm{C}-\mathrm{mol}^{-1}$ \\
\hline$v_{\mathrm{NADH}_{2}-\mathrm{O}_{2}}$ & & 2 & - \\
\hline$K$ & 90 & 110 & $\mu \mathrm{mol}_{h \nu} \mathrm{m}^{-2} \mathrm{~s}^{-1}$ \\
\hline$G_{\mathrm{c}}$ & 1.5 & 10 & $\mu \mathrm{mol}_{h \nu} \mathrm{m}^{-2} \mathrm{~s}^{-1}$ \\
\hline
\end{tabular}




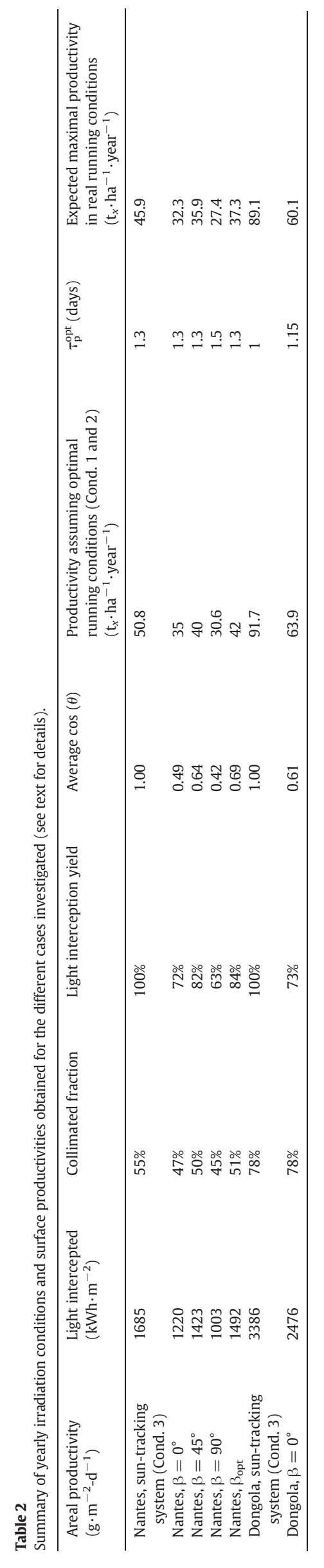

strategies, contamination, etc.) resulting in non-ideal production conditions.

Influence of location was investigated by considering two cases, namely Dongola and Nantes which give very different irradiation conditions in terms of energy received, incident angle, and directdiffuse distribution (see Pruvost et al. [18] for details). Once the illumination conditions are defined, assuming optimal running conditions (luminostat regime and no biomass loss during the night, i.e., Conditions 1 and 2 in Appendix A) makes it possible to calculate the maximal productivity achievable for the actual PBR for a given species at the considered location. This value, which will be lower by definition than the ideal one (i.e., optimal running for ideal irradiation conditions), will set the upper limit for the considered PBR technology at the considered location (only defined by the light use principle-here, surfacelighted-and its ability to collect light as specified in Condition 3). Table 2 reports the results for both locations. As in Pruvost et al. [18], the results emphasize the effect of non-linear photosynthetic conversion efficiency and its direct link with surface productivities. For example, in the case of a horizontal surface-lighted PBR, the 50\% lower light intercepted in Nantes $\left(1220 \mathrm{kWh} \cdot \mathrm{m}^{-2} \cdot\right.$ year $\left.^{-1}\right)$ compared to Dongola leads to only a $40 \%$ lower productivity, as maximal biomass productivity was $35 \mathrm{t}_{x} \cdot$ ha $^{-1} \cdot$ year $^{-1}$ in Nantes versus $60 \mathrm{t}_{x} \cdot \mathrm{ha}^{-1} \cdot$ year $^{-1}$ in Dongola. Note that the value predicted for Dongola confirms that this location leads to near-ideal irradiation conditions, as almost the same productivity was obtained for Dongola and for ideal irradiation conditions.

As light interception over the year is highly dependent on PBR inclination, various inclinations were simulated (Table 2). A $45^{\circ}$ inclination roughly corresponds to the latitude of France and so to the inclination maximizing yearly collected light (i.e., $16 \%$ increase compared to the horizontal case). This results in a $12 \%$ increase in productivity $\left(40 \mathrm{t}_{x} \cdot \mathrm{ha}^{-1} \cdot \mathrm{year}^{-1}\right)$. Although commonly engineered in practice (vertical airlift PBR), vertical inclination $\left(\beta=90^{\circ}\right)$ leads to the lowest light interception, at $40 \%$ lower than a $45^{\circ}$ inclination and $20 \%$ lower than a horizontal system. The resulting maximal productivity is then $30 \mathrm{t}_{x} \cdot \mathrm{ha}^{-1} \cdot \mathrm{year}^{-1}$, i.e., $25 \%$ lower than a $45^{\circ}$ inclination $\left(40 \mathrm{t}_{x} \cdot \mathrm{ha}^{-1} \cdot \mathrm{year}^{-1}\right)$ and $16 \%$ lower than with horizontal systems $\left(35 \mathrm{t}_{x} \cdot\right.$ ha $^{-1} \cdot$ year $\left.^{-1}\right)$.

Previous results were obtained for a fixed inclination angle over the whole year. As an alternative solution, inclination angle can be adjusted monthly to optimize light interception to variation in the sun's azimuth angle over the year [17]. As shown in Table 2 ( $\beta=\beta_{\text {opt }}$ ), there is little justification for such an optimization, as the productivity is found to be close to that achieved at a fixed $45^{\circ}$ inclination angle, yielding only a $5 \%$ increase in maximal productivity $\left(42 \mathrm{t}_{x} \cdot \mathrm{ha}^{-1} \cdot \mathrm{year}^{-1}\right)$. Finally, in the specific case of a sun-tracking system which, by definition, maximizes light interception over time (yearly capture of $1684 \mathrm{kWh} \cdot \mathrm{m}^{-2}$ ), a maximal productivity of $50 \mathrm{t}_{\mathrm{x}} \cdot \mathrm{ha}^{-1} \cdot \mathrm{year}^{-1}$ is achieved in Nantes, which is $25 \%$ higher than with a $45^{\circ}$ inclination (yearly capture of $1423 \mathrm{kWh} \cdot \mathrm{m}^{-2}$ ) and $40 \%$ higher than with a horizontal system (yearly capture of $1220 \mathrm{kWh} \cdot \mathrm{m}^{-2}$ ).

3.3. Realistically achievable productivity of an actual fixed surface-lighted PBR

As described previously, the productivity actually achieved by a realworld PBR is affected by an array of parameters. Our model only refers to light limitation (optimal pH and temperature conditions, no nutrient limitation including by carbon source). Even in this case, expected areal productivity will lower than maximal areal productivity, as discussed in Pruvost et al. [18], due to both the influence of night-time hours (biomass loss) and the transient PBR response imposed by the combined result of both biological kinetics and day-night cycles (biological kinetics being significantly lower than day-night cycle variations). Consequently, light attenuation conditions (luminostat) are never optimal, and light 

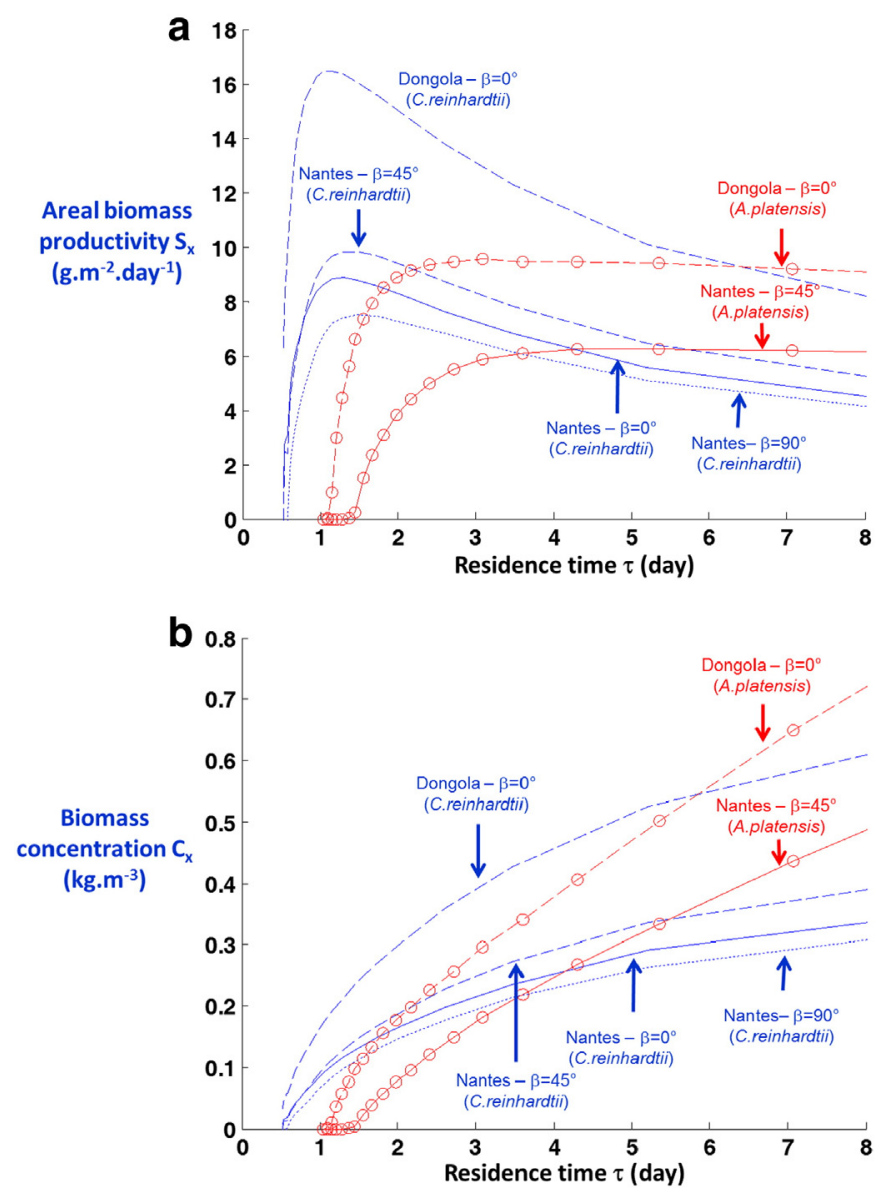

Fig. 2. Yearly average areal productivity (Fig. 2-a) and corresponding biomass concentration (Fig. 2-b) of a horizontal PBR as a function of residence time applied on the cultivation system operated in continuous mode, for Dongola (horizontal system, $\beta=0^{\circ}$ ) and Nantes locations (horizontal system, $\beta=0^{\circ}$; inclined system, $\beta=45^{\circ}$; vertical system, $\beta=90^{\circ}$ ). Values obtained for the cyanobacteria A. platensis (circles) are also added [18].

transmission and/or dark volume occurs. This aspect was extensively investigated in Pruvost et al. [18]. PBR operation was found to be a highly relevant factor, especially harvesting strategy which has a direct influence on the time-course of biomass concentration (Eq. (8)), and thus light attenuation conditions and the resulting photosynthetic conversion. The aim was then to find the best trade-off to achieve maximum biomass productivity over the year of operation. One conclusion of our previous work [18] was that the trade-off was simple to find for cyanobacteria due to the negligible influence of dark volume on the resulting cyanobacterial growth, which made it possible to apply high light attenuations conditions with little effect on biomass productivity.

Harvesting strategy is defined by the harvesting period and the culture volume harvested per day, as represented by the residence time $\tau_{\mathrm{p}}$ (or dilution rate $\mathrm{D}=1 / \tau_{\mathrm{p}}$ ) thus applied. For simplification, only continuous harvesting was considered here (a comparison with semicontinuous mode can be found in Pruvost et al. [17]). Annual average productivity was determined as a function of residence time $\tau_{\mathrm{p}}$ applied. Fig. 2-a reports the biomass productivity results for both the Nantes and Dongola locations, with the corresponding biomass concentration values given in Fig. 2-b. Results obtained in Pruvost et al. [18] for the cyanobacteria $A$. platensis (same irradiations conditions) are also added for comparison. Numerical results are reported in Table 2 (i.e., 'expected maximal productivity in real operating conditions').

These results clearly show that there is an optimal residence time value (noted $\tau_{\mathrm{p}}^{\mathrm{opt}}$ ) and that this value is mainly influenced by PBR location (and to a lesser extent, inclination). When the PBR is operated at its optimal residence time, the maximal expected productivity of the cultivation system is achieved (Table 2). The expected maximal productivities achieved at optimal residence times (realistic operating conditions) were logically systematically lower than the productivities previously obtained assuming optimal operating conditions maintained over the time (hypothetical conditions). A $10 \%$ loss of productivity is observed whatever the case, i.e., a similar decrease to that found for cyanobacteria [18].

It is interesting to compare results between A. platensis and C. reinhardtii. First, as observed previously, $C$. reinhardtii is found to give higher productivity, mainly due to the difference in nitrogen source. However, even if the same type of evolution was achieved for both species at low residence times (rapid decrease in areal productivity towards culture washout for low residence time values, i.e., high dilution rate), high residence time values led to a significant difference in productivity for $C$. reinhardtii whereas $A$. platensis showed relatively little change. Consequently, maximum values of areal productivity values for $C$. reinhardtii were achieved for a very narrow range of residence times, at around $\tau_{\mathrm{p}}^{\mathrm{opt}}=1.3$ days and $\tau_{\mathrm{p}}^{\mathrm{opt}}=1.15$ days for the Nantes and Dongola locations, respectively. This key difference between the two microorganisms will be detailed further, as it has important practical implications, since we concluded it is relatively easy to find a harvesting strategy that maximizes biomass productivity for cyanobacteria, but not for the microalga $C$. reinhardtii.

\subsection{Potential effect of pigment adaption on resulting biomass productivity}

Radiative properties strongly influence light transfer conditions and therefore the resulting biomass productivity. They are directly related to pigment content [31], which is highly variable, especially with solar conditions due to the variability of PFD which is known to directly affect pigment content. This is the so-called "photoacclimation" phenomenon that has been widely investigated in the literature [2,29,37-39]. Numerous parameters were found to influence pigment content in microalgae, and a deep modeling of this phenomenon is out of the scope of this study. In a first approach, we only investigated how acclimation to PFD could affect our predictions of biomass productivities, given that PFD is known to be a major parameter of photoacclimation.

As the reference case, we supposed an adaption of pigment content to the monthly average PFD value, based on the work of Takache et al. [22] where photoacclimation was investigated by growing C. reinhardtii under various PFDs. An additional case was simulated that assumed constant values of radiative properties throughout the year (i.e., radiative properties were calculated for a yearly average PFD value). The corresponding radiative properties for both Dongola and Nantes locations are given in Fig. 3-a. In accordance with Takache et al. [22], increasing PFD leads to lower mass absorption coefficients $\left(E_{\mathrm{a}}\right)$ and higher mass scattering coefficients $\left(E_{\mathrm{s}}\right)$. The different radiation conditions in both Dongola and Nantes result in different evolutions in radiative properties. The highest evolution was found at Nantes when introducing a relation to the monthly average PFD. This is directly related to the high variation in PFD observed over the year at this location.

Fig. 3-b charts the influence on resulting biomass productivity and corresponding biomass concentration for the Nantes location. Surprisingly, despite the significant evolution in radiative properties, the ultimate effect on productivity remains low. In fact, the negligible influence of photoacclimation on resulting productivity is explained by the fact that change in radiative properties is partially offset by the biomass concentration then achieved at a given residence time (Fig. 3-b).

Although the effect of change in pigmentation content on productivity can be neglected in a first instance, accurate radiative properties are of primary relevance if radiative transfer has to be optimized, such as when investigating light attenuation conditions induced in solar conditions (see further). However, specific investigations are needed, as the photoacclimation process should be directly affected by these light attenuation conditions, which would add complex coupling in the process 

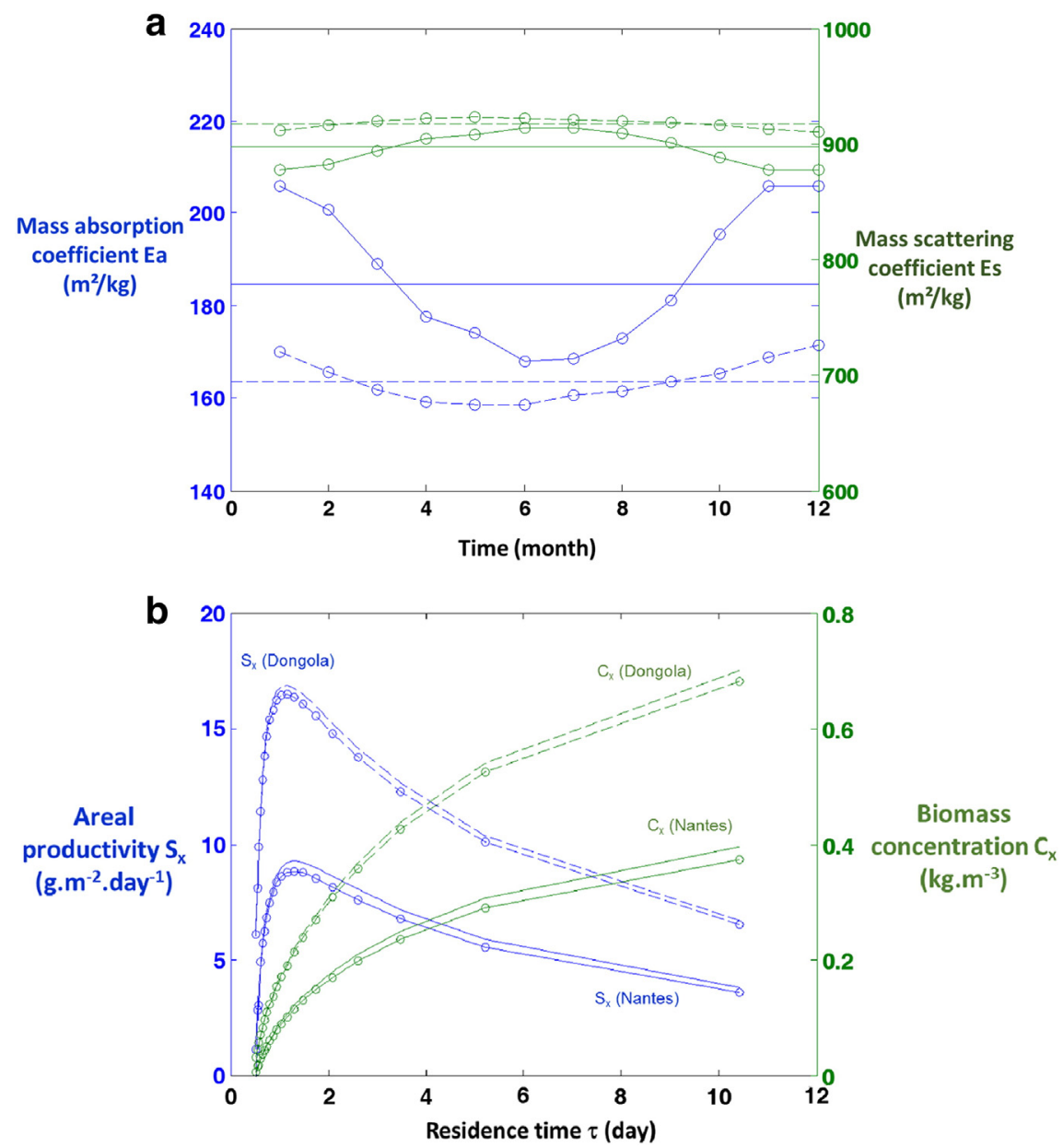

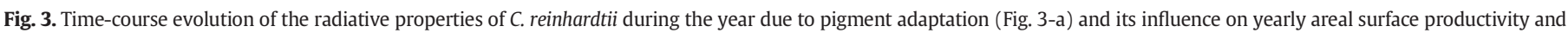

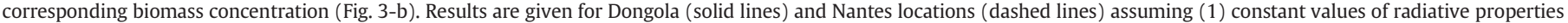
over the entire year (no symbol) and (2) pigment adaption to the monthly average PFD value (circles).

model. In addition, pigment adaptation follows a given kinetics, which is usually assumed to be in the range of several days [37]. At this juncture, then, our results can only be regarded as a very first estimate. Our simulation assumed that a photoacclimation occurs and subsequently evolves with the monthly average PFD value as the best representation proposable given the data currently available.

\subsection{Detailed analysis of a given surface-lightened PBR located in Nantes}

Year-long evolutions in both biomass concentration and areal productivity achieved when applying optimal residence times are given in Fig. 4-a for Nantes and Fig. 4-b for Dongola (monthly average values are also given to help read the figures). The direct relation to annual variation in irradiation conditions is clearly visible, with productivity in Dongola being higher but also evolving less over the year. Values obtained for optimal conditions (Conditions 1, 2 and 3 in Appendix A) were also added, with consequently higher-thanrealistic productivities, even in cases applying optimal residence times.

Fig. 5-a gives results for non-optimal residence time values $\left(\tau_{\mathrm{opt}} / 2\right.$, and $\left.2 . \tau_{\text {opt }}\right)$. As expected, both resulted in lower productivities than that achieved at optimal residence time, but for different reasons. To explain this difference, light transfer conditions have to be analyzed in terms of illuminated light fraction $\gamma$. Fig. 5-b charts the year-long evolution in terms of the corresponding weekly-averaged values (calculated only during the day period, as light fraction is meaningless in nighttime conditions). As already observed with $A$. platensis, optimizing the residence time in the cultivation system is not sufficient to maintain the ideal condition of a luminostat regime $(\gamma=1)$ because the biomass growth rate is not high enough to offset the rapid changes in sunlight intensity. The optimal residence time can only be regarded as the best compromise to achieve maximum biomass productivity over a given cultivation period (here, a full year period).

As shown in Fig. 5-b, increasing the residence time (2. $\tau_{\text {opt }}$ ) leads to a higher biomass concentration in the cultivation system, which then promotes periods when dark zones are encountered $(\gamma<1)$. As a result, biomass productivity was lower throughout the year due to the negative impact of respiration on resulting microalgae growth (Fig. 5-a). Increasing the residence time can prove beneficial in winter due to the seasonally-lower growth rate. As optimal residence time is defined as a trade-off calculated on a whole-year basis, increasing the residence time for this specific period can then have a positive impact on productivity. Our simulations do however reveal that adapting the residence time has only negligible benefit, as it reaches practically the same productivity as for the year-long optimal residence time. This is mainly explained by the fact that winter-season productivity is too low to gain any real benefit from winter-season optimization.

Fig. 5 also presents results for a less-than-optimal residence time $\left(\tau_{\text {opt }} / 2\right)$. For the lower winter irradiations conditions, biomass growth is insufficient, resulting in biomass washout (null productivity). For 

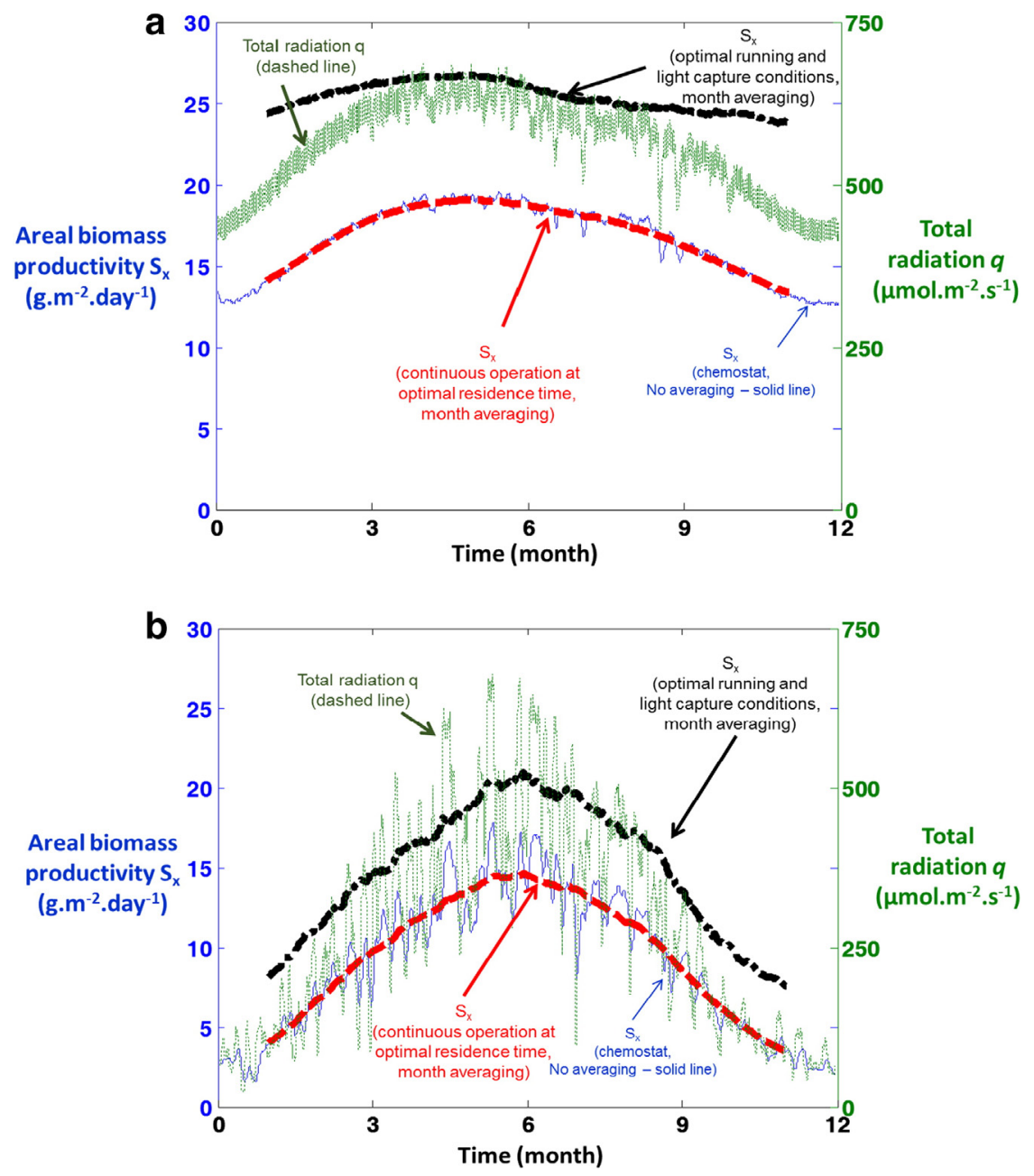

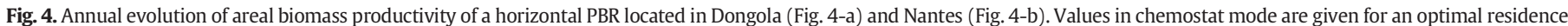

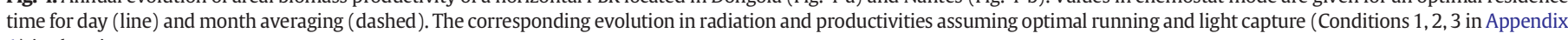
A) is also given.

spring and autumn, growth occurs, but the biomass concentration is too low to obtain full light absorption, which enables light transmission to occur $(\gamma>1)$, resulting in a lower productivity than that achieved at optimal residence time (Fig. 5-a). As a consequence, high near-maximal productivity is only achievable in the summer period.

Results of Fig. 5 tend to argue for a year-long optimization of residence time in a PBR. Modeling proves very valuable for this purpose, as it enables biomass productivity to be calculated for any residence time value. Only fixed values were applied in previous simulations, but time-varying values could easily be considered and could lead to higher expected productivity. This kind of optimization is out of the scope of this study, but as a first estimate, the model was used to calculate the ideal biomass concentration values that should be obtained in the cultivation system to maintain the luminostat regime over time. Once this biomass concentration time-course was known, the model was used to deduce the corresponding residence time. Note that here, we assumed instantaneous adaptation of biomass concentration to any variation in light received at the PBR surface (or, in other words, we supposed an infinitely fast growth kinetics, which obviously cannot be observed in reality due to the significantly lower kinetics of photosynthetic growth compared to sunlight time variation). The corresponding results are given in Fig. 6, which clearly illustrates that the optimal residence time is a compromise over the whole-year duration and, by definition, never corresponds to the ideal value for a given within-year period/season. In the present case, higher values up to $\tau_{\mathrm{p}}=2.3$ days should ideally have been applied in winter, along with lower values down to $\tau_{\mathrm{p}}=0.8$ days in summer.

\subsection{Analysis of light attenuation conditions encountered in a PBR over the year}

Fig. 7-a and b charts the biomass concentration evolutions for two typical days of the year (6th February, winter period, and 12th July, summer period) obtained at the optimal residence time value yielding maximal expected productivity ( $\tau_{\mathrm{p}}^{\mathrm{opt}}=1.3$ day $)$. The daytime evolution of biomass concentration as a result of day-night cycle is plotted. Photosynthetic growth occurs throughout the day, after which biomass concentration decreases during the night due to biomass respiration. A higher growth is achieved in summer, due not just to the higher PFD values but also the longer daylight hours (around $16 \mathrm{~h}$ in summer versus $11 \mathrm{~h}$ in winter). Note that $C$. reinhardtii shows a higher biomass concentration than $A$. platensis but also a higher range of evolution over the $24 \mathrm{~h}$ period. $C$. reinhardtii demonstrates not only higher growth during the day but also higher biomass consumption during the night. This is fully explained by the higher quantum yield (on ammonium as $\mathrm{N}$ source) of $C$. reinhardtii at light, and by the significant difference in specific biomass decay rate during the night between eukaryotes and prokaryotes, as already observed by Le Borgne \& Pruvost [26], with $C$. reinhardtii showing a 4-fold higher specific biomass decay rate. The 

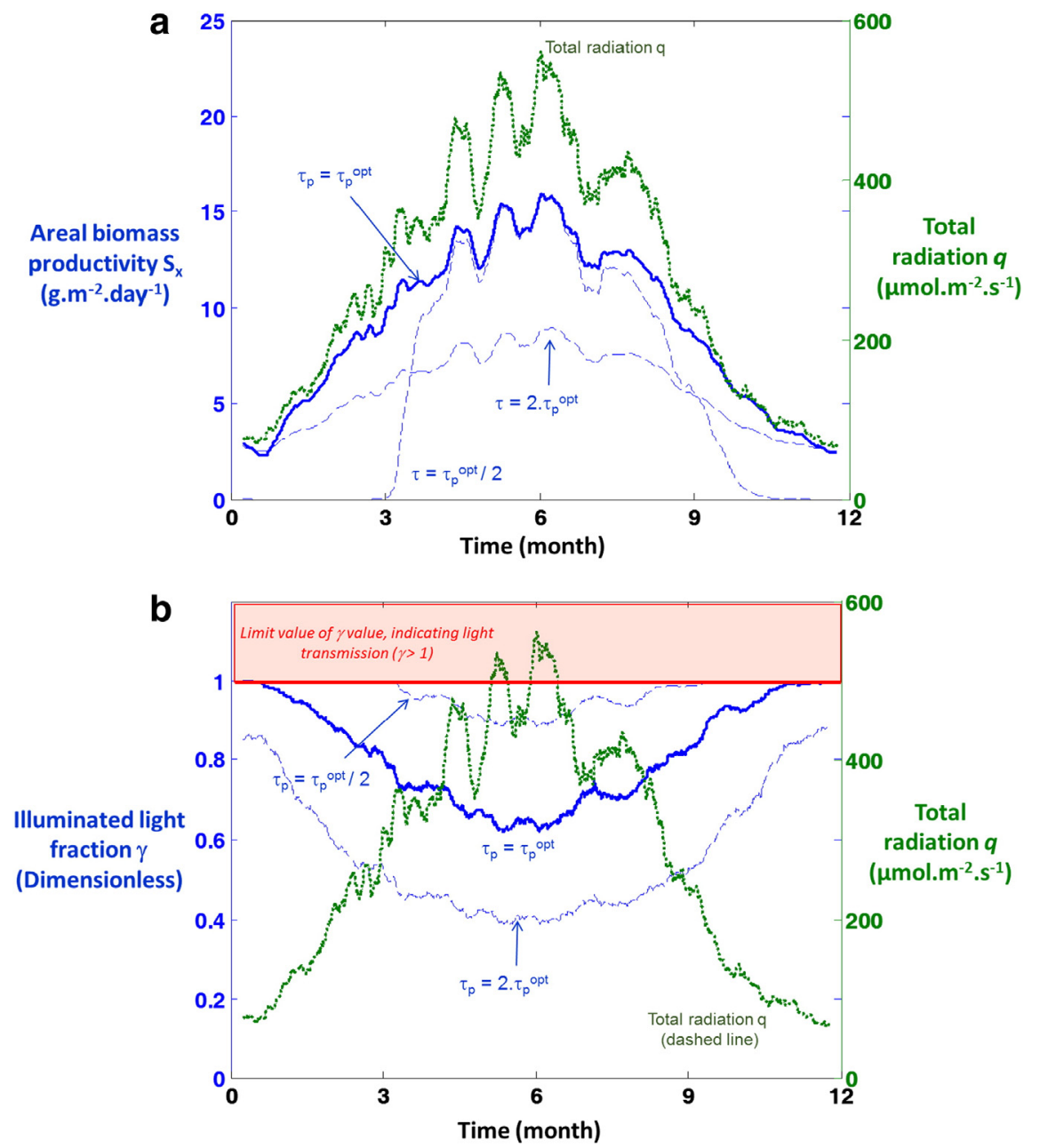

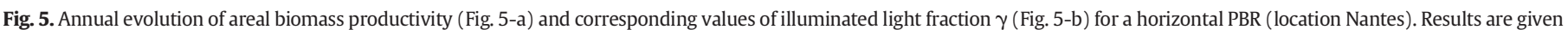

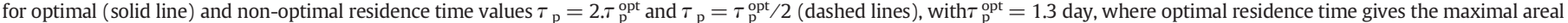
productivity (weekly averaged values are represented here for clearer graphic illustration).

net result is that $C$. reinhardtii shows a higher range of variation in biomass concentration.

As a direct consequence of the dynamics induced by day-night cycles, light attenuation conditions in the cultivation system are not constant. Photosynthetic microorganisms thus encounter specific light attenuation conditions which are the result of PFD variation onto the PBR surface and light absorption conditions in the culture volume. As shown previously, the illuminated light fraction $\gamma$ can be a useful value to represent light attenuation conditions, due to its direct relation to the resulting photosynthetic conversion and biomass productivity of

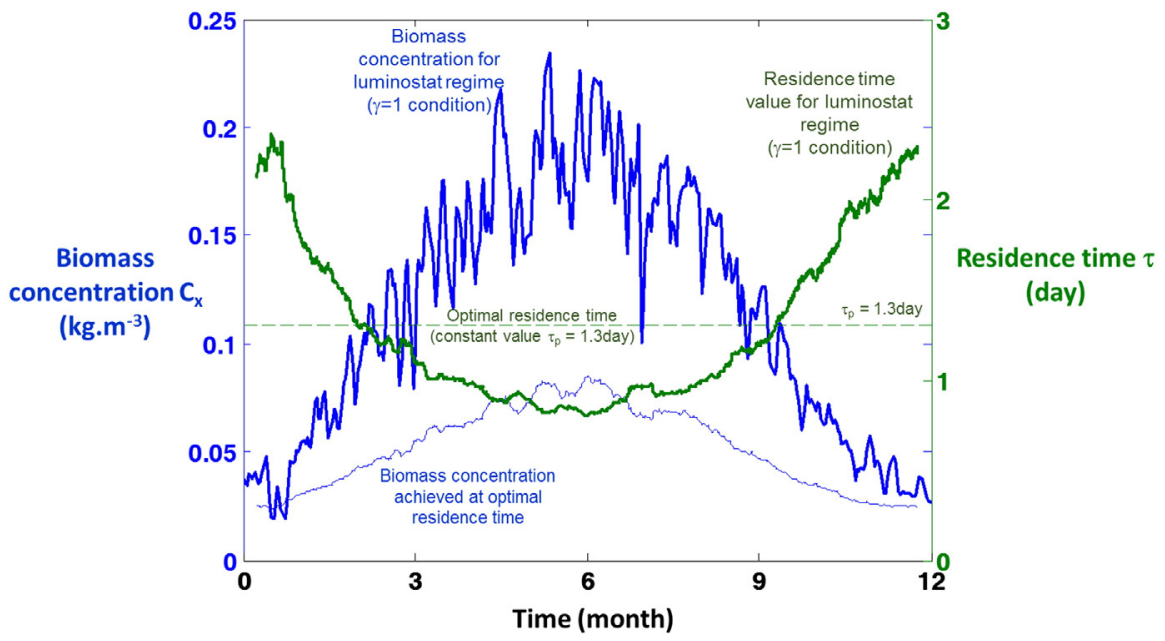

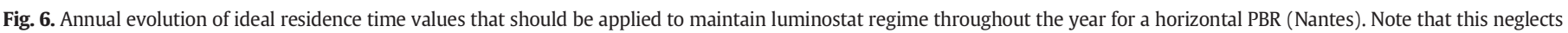
biomass growth kinetics and consequently instantaneous process adaptation to illumination conditions (see text for details). 

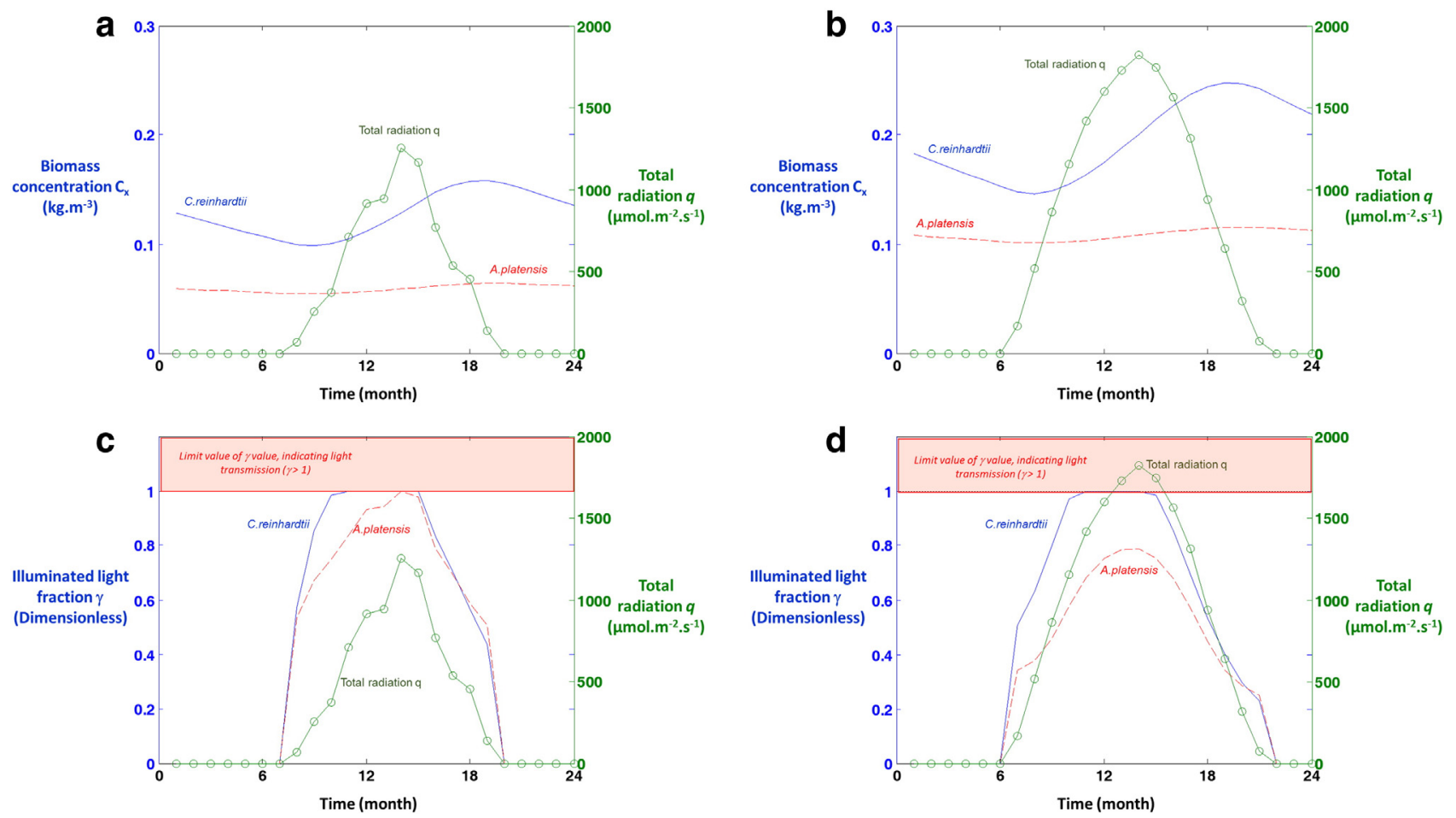

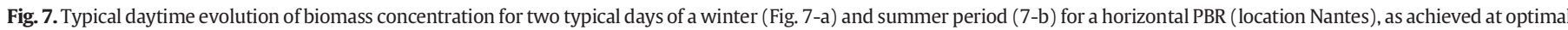

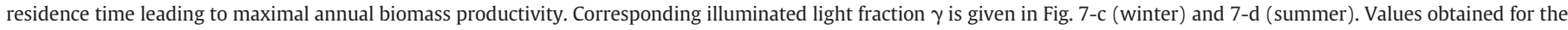
cyanobacteria A. platensis (dashed line) are also added (Pruvost et al., 2012).

the cultivation system. This value was then retained in a first instance to represent the light attenuation conditions encountered. Values obtained for the two previous typical days are given in Fig. 7-c and d, which emphasize the huge daytime variation in terms of light attenuation conditions, with a rapid increase in illuminated light fraction immediately after sunrise up to maximal values at noon, followed by a rapid drop down to null value at sunset. It is again interesting to compare results between $C$. reinhardtii and $A$. platensis. First, due to its higher evolution in biomass concentration during both night and day, C. reinhardtii experienced a more marked evolution in light attenuation conditions compared to A. platensis. This is reinforced by the result of the optimization procedure which led to different residence times needed to maximize annual productivity for $C$. reinhardtii ( $\tau_{\mathrm{opt}}=1.3$ day) and

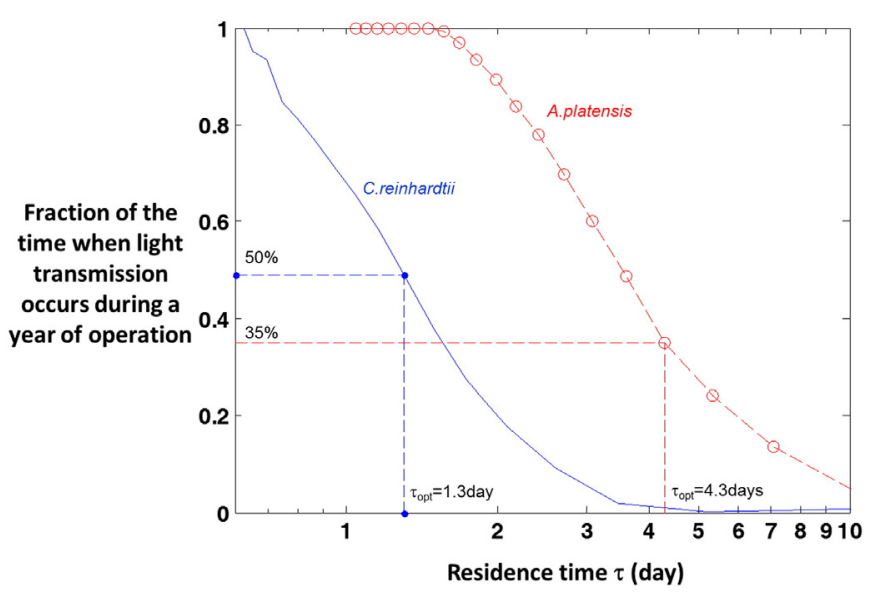

Fig. 8. Distribution of the number of hours per year when light transmission $(\gamma>1)$ occurs, as a function of residence time applied in the cultivation system, for both $C$. reinhardtii (solid line) and A. platensis (circles). Values are normalized with respect to total number of illuminated hours in the year, i.e., $4355 \mathrm{~h}$ for the Nantes location.
A. platensis ( $\tau_{\mathrm{opt}}=4.3$ days), thus leading to different biomass concentration and light attenuation conditions, as discussed below.

For the cyanobacterium A. platensis, due to the negligible effect of dark volume on resulting productivity, optimization procedure leads to a low illuminated fraction value (as achieved at high residence time), with light attenuation conditions mainly composed of full-light attenuation conditions with dark volumes. Note however that the highest illuminated fraction values were obtained in winter, with possible light transmission $(\gamma>1)$ at noon, despite the lower PFD than in summer. This was already observed in Pruvost et al. [17] and was explained by the particular day-night cycles encountered in winter at the Nantes location. Lower irradiation in winter results in a lower biomass concentration, and the sharp peaks in PFD with short periods of illumination found in winter mean that light transmission can occur, especially on bright days at noon.

For the microalga $C$. reinhardtii, the optimization procedure tends to minimize periods when dark volumes are encountered due to their negative effect on eukaryotic cell growth. As a result, lower light attenuation conditions and subsequently higher $\gamma$ values are promoted. In fact, periods of several hours with light transmission $(\gamma>1)$ are encountered in both the summer and winter periods. Light attenuation conditions resulting from the optimization procedure are ultimately highly different between microalga and cyanobacteria, both in terms of kinetics and the range of $\gamma$ values covered. This is confirmed in Fig. 8 where the fraction of the time when light transmission is non-zero is represented as a function of residence time, the fraction being defined as a function of illuminated hours in the year, i.e., $4355 \mathrm{~h}$ for Nantes location. Fig. 8 indicates that residence time has a strong influence on the number of hours when light transmission occurs as it is directly dependent on biomass concentration. For example, a long residence time results in a large biomass concentration and strong PFD attenuation. However, evolutions in biomass productivity and light transmission with residence time differ between species. For A. platensis, when applying the optimal residence time, the cultivation systems are found to be operated with around 35\% of illuminated hours with light transmission, versus up to 
$50 \%$ for $C$. reinhardtii. This analysis underlines a relevant feature of microalgae solar production. For cyanobacteria, it proved fairly easy to work close to maximal performance of the cultivation system with appropriate optimization of residence time, whereas for microalgae, residence time values maximizing productivity are (1) in a narrower range and (2) promote low light attenuation conditions with light transmission. This is related to the well-known fact that photosynthetic conversion, and more generally metabolism of photosynthetic microorganisms, is highly affected by an excess of light. This could induce specific biological responses, such as pigment decreases or even photoinhibition which could induce culture drift or loss of efficiency and potentially impairs culture stability $[2,9,21,40-43]$. Such aspects were not considered in our model, as it would certainly imply a very complex formulation. Photoinhibition is for example the result of not just light received but also duration of exposure to over-saturating light, etc.... Deeper investigation is needed, but based on our results, we can tentatively conclude that the photoinhibition (or related phenomena) countermeasure usually applied in practice, i.e., to increase biomass concentration to induce what is termed "mutual self-shading" [43-45], will tend to decrease the resulting productivity in microalgae cultivation due to a higher impact of the dark volumes thus generated. This clearly points to the need to find a compromise between maximizing biomass productivity and maintaining stable production conditions by setting the appropriate residence time and associated biomass concentration in the cultivation system. For example, if residence time is doubled in a C. reinhardtii system, the PBR presents non-zero light transmission during only $15 \%$ of illuminated hours, which can be considered acceptable as a first-order approximation as it translates into only a limited loss of productivity (about 10-15\%). Modeling is especially useful here, as it can predict the evolution of parameters defining the state of the culture so as to determine the optimum operating conditions leading to both robust and efficient production.

\section{Conclusions}

Because of their respiration activity in light, microalgae are known to have a high sensitivity to high light attenuation conditions, dark volumes in PBR leading then to a decrease in biomass productivity. The aim was to investigate consequences of this behavior in the specific case of changing light conditions of a solar use. This was applied to the growth of the microalga $C$. reinhardtii (ammonium as $\mathrm{N}$-source) in a surface-lighted PBR.

By applying the concept of ideal PBR, an ideal productivity of roughly $60 \mathrm{t}_{x}$ ha $^{-1} \cdot$ year $^{-1}$ was obtained (and $100 \mathrm{t}_{x}$ ha $^{-1} \cdot$ year $^{-1}$ with suntracking). As any change in location modifies the irradiation conditions, simulations were conducted for Dongola in Sudan and Nantes in France for optimal and realistic operating conditions. Comparison against the cyanobacterium $A$. platensis emphasized the relevance of non-optimal light attenuation conditions as a factor shaping the resulting productivity. In addition to light transmission, which leads to a loss of productivity whatever the species, the appearance of dark zones has an especially negative influence in microalgae cultivation settings. As a result, biomass concentration was found to be a parameter of primary relevance. Modeling proved extremely useful for relating growth (biomass concentration), radiative transfer and solar radiation conditions. It was used to optimize the residence time applied in the cultivation system so as to find the best trade-off in terms of biomass concentration that will maximize, on a given period (full year here), solar conversion in the culture bulk and thus the resulting biomass productivity. This procedure was applied in various cases, emphasizing for example the relation between PBR location and inclination on resulting productivity. As a typical example, our figures point to a maximum productivity of around $40 \mathrm{t}_{x} \mathrm{ha}^{-1} \cdot$ year $^{-1}$ achievable at Nantes (France) with C. reinhardtii.

Finally, we investigated the consequence of low light attenuationpromoting optimization procedure applied to microalgae cultivation to safeguard against the marked negative influence of dark volume on microalgae growth. By simulating PBR operation over a whole-year period, we analyzed the consequences of such a result in terms of light attenuation conditions encountered during the year. A key finding was the increased risk of receiving over-saturating light, which could impair PBR stability in solar conditions in cases where high PFDs are combined with low biomass concentrations. We found that the fast kinetics of radiation conditions compared to photosynthetic growth makes this fact difficult to avoid, especially when applying conditions tending towards maximal productivity.

\begin{tabular}{|c|c|}
\hline \multicolumn{2}{|c|}{ Nomenclature } \\
\hline A & local specific radiant energy absorbed $\left[\mu \mathrm{mol} \cdot \mathrm{s}^{-1} \cdot \mathrm{kg}_{\mathrm{x}}^{-1}\right]$ \\
\hline$a_{\text {light }}$ & specific illuminated area for the photobioreactor $\left[\mathrm{m}^{-1}\right]$ \\
\hline$b$ & back-scattered fraction for radiation [dimensionless] \\
\hline$C_{X}$ & biomass concentration $\left[\mathrm{kg}_{\mathrm{x}} \cdot \mathrm{m}^{-3}\right]$ \\
\hline$D$ & dilution rate $\left[\mathrm{s}^{-1}\right]$ \\
\hline$E_{\mathrm{a}}$ & mass absorption coefficient $\left[\mathrm{m}^{2} \cdot \mathrm{kg}_{\mathrm{x}}^{-1}\right]$ \\
\hline$E_{\mathrm{s}}$ & mass scattering coefficient $\left[\mathrm{m}^{2} \cdot \mathrm{kg}^{-1}\right]$ \\
\hline$G$ & local spherical irradiance $\left[\mu \mathrm{mol} \cdot \mathrm{s}^{-1} \cdot \mathrm{m}^{-2}\right]$ \\
\hline$G_{\mathrm{c}}$ & compensation irradiance value $\left[\mu \mathrm{mol} \cdot \mathrm{s}^{-1} \cdot \mathrm{m}^{-2}\right]$ \\
\hline $\mathrm{J}_{\mathrm{O} 2}$ & $\begin{array}{l}\text { specific rate of } \mathrm{O}_{2} \text { production or consumption } \\
{\left[\mathrm{mol}_{\mathrm{O} 2} \cdot \mathrm{kg}^{-1} \cdot \mathrm{s}^{-1}\right]}\end{array}$ \\
\hline$J_{\mathrm{NADH}}$ & $\begin{array}{l}\text { specific rate of cofactor regeneration on the respiratory chain } \\
{\left[\mathrm{mol}_{\mathrm{NADH}_{2}} \mathrm{~kg}_{\mathrm{X}}^{-1} \mathrm{~s}^{-1}\right]}\end{array}$ \\
\hline K & half saturation constant for photosynthesis $\left[\mu \mathrm{mol} \cdot \mathrm{s}^{-1} \cdot \mathrm{m}^{-2}\right]$ \\
\hline $\mathrm{K}_{\mathrm{r}}$ & respiration inhibition constant $\left[\mu \mathrm{mols} \cdot{ }^{1} \cdot \mathrm{m} \cdot{ }^{2}\right]$ \\
\hline$L$ & depth of the rectangular photobioreactor [m] \\
\hline$M_{X}$ & $\mathrm{C}$-molar mass for the biomass $\left[\mathrm{kg}_{\mathrm{X}} \cdot \mathrm{mol}_{\mathrm{X}}^{-1}\right]$ \\
\hline$q$ & $\begin{array}{l}\text { photon flux density on a given surface (PFD) } \\
{\left[\mu \mathrm{mol} \cdot \mathrm{s}^{-1} \cdot \mathrm{m}^{-2}\right]}\end{array}$ \\
\hline$r_{\mathrm{X}}$ & biomass volumetric growth rate (productivity) $\left[\mathrm{kg}_{\mathrm{x}} \cdot \mathrm{m}^{-3} \cdot \mathrm{s}^{-1}\right]$ \\
\hline$S_{\mathrm{L}}$ & illuminated surface of the photobioreactor $\left[\mathrm{m}^{2}\right]$ \\
\hline$S_{X}$ & areal biomass productivity $\left[\mathrm{kg}_{\mathrm{X}} \cdot \mathrm{m}^{-2} \cdot \mathrm{s}^{-1}\right]$ \\
\hline$t$ & time $[s]$ \\
\hline$V_{\mathrm{r}}$ & photobioreactor volume $\left[\mathrm{m}^{3}\right]$ \\
\hline$x_{d}$ & diffuse fraction for incident PFD at any location [-] \\
\hline & epth of culture [m] \\
\hline
\end{tabular}

\section{Greek letters}

$\alpha \quad$ linear scattering modulus [dimensionless]

$\beta \quad$ inclination of the photobioreactor surface [rad]

$\gamma \quad$ fraction for working illuminated volume in the photobioreactor [dimensionless]

$\delta \quad$ extinction coefficient for the two-flux method $\left[\mathrm{m}^{-1}\right]$.

$\theta \quad$ incident angle (defined from the outward normal of the PBR) [rad]

$\rho_{\mathrm{M}} \quad$ maximum energy yield for photon conversion [dimensionless]

$v_{\mathrm{O}_{2}-X} \quad$ stoichiometric coefficient of the oxygen production $\left[\mathrm{mol}_{\mathrm{O}_{2}}\right.$. $\mathrm{mol}_{\mathrm{X}}^{-1}$ ]

$v_{\mathrm{NADH}_{2}-\mathrm{O}_{2}}$ stoichiometric coefficient for the cofactor regeneration of the respiratory chain $\left[\mathrm{mol}_{\mathrm{NADH}_{2}} \cdot \mathrm{mol}_{\mathrm{O}_{2}}^{-1}\right]$

$\tau_{p} \quad$ hydraulic residence time $[s]$

$\overline{\phi_{\mathrm{O}_{2}}^{\prime}} \quad$ mole quantum yield for the Z-scheme of photosynthesis $\left[\mathrm{mol}_{\mathrm{O}_{2}}, \mu \mathrm{mol}_{\mathrm{hv}}^{-1}\right]$

\section{Subscripts}

// related to direct radiation

$\perp \quad$ related to normal direct radiation

$\cap \quad$ related to total diffuse radiation

col related to the collimated fraction of irradiance

dif related to the diffuse fraction of irradiance

dark related to a dark zone in the photobioreactor

light related to an illuminated zone in the photobioreactor

max related to maximum available solar radiation

opt related to the optimal value for residence time 
Other

$<X>=\frac{1}{V} \iiint_{V} X d V$ spatial averaging

$\bar{X}=\frac{1}{\Delta t} \int_{\Delta t} X \mathrm{~d} t$ time averaging

Abbreviations

PAR photosynthetically active radiation

PBR photobioreactor

PFD photon flux density

\section{Acknowledgments}

This work was supported by the French National Research Agency projects DIESALG (ANR-12-BIME-0001-02) and BIOSOLIS (ANR-07BIOE-007). This is also a part of the research activities conducted on AlgoSolis R\&D facility (http://www.algosolis.com). This work has been sponsored by the french governement program "investissements d'avenir" through the IMobS3 laboratory of excellence (ANR-10-LABX16-01).

\section{Appendix A}

\section{A.1. Description of the photobioreactor model}

In the specific case of outdoor biomass production, numerous features can impair process production, such as mineral or carbon limitation, nonideal temperature or $\mathrm{pH}$ control, non-optimized harvesting strategies, contamination, etc. Our model is voluntarily restricted to the case of the so-called light-limited regime, where only light limits growth. This makes process productivity solely dependent on light capture and use in the culture volume. As light is the only limiting factor, the maximal performances of a given PBR can be calculated at a given location and for a given species. Based on this assumption, we had already proposed a model for solar PBR which can be directly applied in this study. As it is already described elsewhere [17-19], only main features are reported here.

The model applies to cultivation systems presenting a flat illuminated surface (ponds, rectangular PBR, etc.). The one-dimensional and azimuth-independence assumptions can then be used to describe the irradiance field in the culture bulk, making it possible to apply the twoflux radiative model with its corresponding analytical solutions [46]. Application to the solar case implies taking into account non-normal incidence (thus introducing the incident angle $\theta$ ) with a separate treatment of the direct and diffuse components of the radiation due to their difference in angular distribution on the PBR surface [17]. The total hemispherical incident light flux density (or PFD, see next section) $q$ is divided into the direct $q_{/ /}$and diffuse $q_{\cap}$ components $\left(q=q_{/ /}+q_{\cap}\right.$ ). Total irradiance (representing the amount of light received in the culture bulk) is given by summing the resulting contribution of collimated and diffuse radiation:

$G(z)=G_{\text {col }}(z)+G_{\text {dif }}(z)$

where $G_{\mathrm{col}}$ is the irradiance field for collimated radiation, as given by:

$\frac{G_{\mathrm{col}}(z)}{q_{/ /}}=\frac{2}{\cos \theta} \frac{(1+\alpha) \exp \left[-\delta_{\mathrm{col}}(z-L)\right]-(1-\alpha) \exp \left[\delta_{\mathrm{col}}(z-L)\right]}{(1+\alpha)^{2} \exp \left[\delta_{\mathrm{col}} L\right]-(1-\alpha)^{2} \exp \left[-\delta_{\mathrm{col}} L\right]}$

and $G_{\text {dif }}$ the irradiance field for diffuse radiation:

$\frac{G_{\mathrm{dif}}(z)}{q_{\cap}}=4 \frac{(1+\alpha) \exp \left[-\delta_{\mathrm{dif}}(z-L)\right]-(1-\alpha) \exp \left[\delta_{\mathrm{dif}}(z-L)\right]}{(1+\alpha)^{2} \exp \left[\delta_{\mathrm{dif}} L\right]-(1-\alpha)^{2} \exp \left[-\delta_{\mathrm{dif}} L\right]}$.

In these equations, $\alpha=\sqrt{\frac{E_{a}}{\left(E_{a}+2 b E_{s}\right)}}$ is the linear scattering modulus, and $\delta_{\text {col }}=\frac{\alpha C_{X}}{\cos \theta}\left(E_{\mathrm{a}}+2 b E_{\mathrm{s}}\right)$ and $\delta_{\mathrm{dif}}=2 \alpha C_{x}\left(E_{\mathrm{a}}+2 b E_{\mathrm{s}}\right)$ are the twoflux collimated and diffuse extinction coefficients, respectively. $E_{\mathrm{a}}$ is mass absorption coefficient and $E_{\mathrm{s}}$ mass scattering coefficient for the cultivated photosynthetic microorganism, $b$ the back-scattered fraction, and $C_{X}$ the biomass concentration in the culture medium. Here, radiative properties $\left(E_{\mathrm{a}}, E_{\mathrm{s}}, \mathrm{b}\right)$ were spectrally averaged over the PAR. Values are given in Table 1.

Once the irradiance field is known (Eq. (1)), the illuminated fraction $\gamma$, a relevant parameter in PBR engineering, can be deduced $[17,23,34$, 35]. Schematically, the culture volume $V_{\mathrm{r}}$ is split into two zones: an illuminated zone of volume $V_{\text {light }}$ and a dark zone of volume $V_{\text {dark. }}$. The illuminated fraction $\gamma$ is then given by the depth of culture $z_{\mathrm{c}}$ where the irradiance of compensation $G\left(z_{\mathrm{c}}\right)=G_{\mathrm{c}}$ is obtained, where $G_{\mathrm{c}}$ is the minimum value of radiant energy required to obtain a positive photosynthetic growth. In the case of cultivation systems with one-dimensional light attenuation, we obtain:

$\gamma=\frac{V_{\text {light }}}{V_{\mathrm{r}}}=\frac{z_{\mathrm{c}}}{L}$

where $L$ is PBR depth, with $G_{\mathrm{c}}=10 \mu \mathrm{mole} \cdot \mathrm{m}^{-2} \cdot \mathrm{s}^{-1}$ for $C$. reinhardtii [35], and $G_{\mathrm{c}}=1.5 \mu \mathrm{mole} \cdot \mathrm{m}^{-2} \cdot \mathrm{s}^{-1}$ for A. platensis [35]. A $\gamma$ value below 1 indicates that all light available for photosynthesis is absorbed by the culture. Conversely, when the illuminated fraction is greater than 1 (a hypothetical representation because at maximum $V_{\text {light }}=$ $V_{\mathrm{r}}$ ), some of the light is transmitted (the so-called 'kinetic regime').

Determining the irradiance field makes it possible to determine the corresponding local photosynthetic growth rate in the culture volume, as obtained from the growth kinetic relations presented in section 2.b (Modeling photosynthetic growth for cyanobacteria and microalgae). The mean biomass volumetric growth rate is then obtained by averaging the local formulation of the volumetric growth rate over the culture volume. For a cultivation system with one-dimensional light attenuation as in our study, this consists in a simple integration along the depth of culture $z$.

$\left\langle r_{X}\right\rangle=\frac{1}{L} \int_{z=0}^{z=z_{L}} r_{X} \mathrm{~d} z$,

Finally, the determination of the mean growth rate allows the mass balance equation, here for biomass, to be solved $[11,47,48]$. For a continuous system assuming perfectly mixed conditions, this equation is:

$\frac{\mathrm{d} C_{X}}{\mathrm{~d} t}=\left\langle r_{X}\right\rangle-\frac{C_{X}}{\tau_{\mathrm{p}}}$

where $<r_{x}>$ is the mean biomass volumetric growth rate in the system, and $\tau_{\mathrm{p}}$ the residence time resulting from the liquid flow rate of the feed (fresh medium).

The variable PFD in sunlight conditions means that the irradiance field inside the culture bulk and the resulting local and mean volumetric growth rates vary continuously, and hence steady-state cannot be assumed in Eq. (A6) This implies solving the transient form of the mass balance equation (using for example the ode23tb routine in Matlab software).

Finally, having determined the time-course of biomass concentration, we can now calculate the corresponding biomass productivity. Surface productivity $P_{S}\left(\mathrm{~g} \cdot \mathrm{m}^{-2}\right.$. day $\left.{ }^{-1}\right)$ will be used here as a useful variable to extrapolate to land area production, as given by:

$P_{S}=\frac{C_{x} V_{\mathrm{r}}}{\tau_{\mathrm{p}} S_{\text {light }}}=\frac{C_{x}}{\tau_{\mathrm{p}} a_{\text {light }}}$

A.2. Definition of the ideal operating conditions for a solar surface-lighted $P B R$

The model can be usefully introduced with specific conditions related to cultivation system operation and its light capture in order to investigate their effect on process performances to gain deeper insight into process behavior, and to introduce the concept of ideal reactor to 
calculate the theoretical limit in terms of productivity. The concept of ideal reactor requires the following conditions to be met [18]:

(i) Luminostat regime ('Condition 1'): optimal light absorption conditions inside the culture are maintained throughout the day (see [32]). For prokaryotic cells, this only implies full light absorption $(\gamma \leq 1)$. For eukaryotic cells, owing to respiration in light, the condition of no dark zone in the bulk culture would have to be added $(\gamma=1)$. As a common constraint of both organisms, only the $\gamma=$ 1 condition defining the luminostat regime can be retained (same results for cyanobacteria with $\gamma \leq 1$ ). Obviously, no limitation other than light occurs (no mineral or carbon limitation, optimal temperature and $\mathrm{pH}$ conditions).

(ii) Ideal biological response ('Condition 2'): there are no adverse effects of strong light on photosynthetic conversion (no photoinhibition) for cells exposed to short bursts of high irradiances. Only the inevitable progressive light saturation of photosynthesis occurs. Finally, it is assumed that there is no night-time biomass loss due to respiration.

In addition, as fixed PBRs are unable to collect all the available solar energy due to the sun changing position, we introduced a condition-set for optimal capture, assuming that maximal solar radiation is hypothetically collected at each time of the day ('Condition 3'). This means that all the radiation available is assumed to enter the PBR with a direct $q_{\perp}$ component at normal incidence $(\theta=0)$. Meeting this optimal capture condition means tracking the sun throughout the day, i.e., having a moving PBR. This condition may clearly be an unrealistic technical option for most surface-lighted PBRs, but it does give maximal light capture. This condition was thus deliberately set apart from the two others. All three conditions can be easily introduced into the model (see [18]).

\section{References}

[1] C. Posten, C. Walter, Microalgal Biotechnology: Potential and Production, Walter de Gruyter \& Co, 2012.

[2] A. Richmond, Handbook of Microalgal Culture: Biotechnology and Applied Phycology, Blackwell Sciences Ltd, Oxford, UK, 2004.

[3] J.R. Benemann, Hydrogen and methane production by microalgae, in: B.S. Ltd (Ed.), Handbook of Microalgal Culture: Biotechnology and Applied Technology, A.Richmond, 2004.

[4] Y. Chisti, Biodiesel from microalgae, Biotechnol. Adv. 25 (2007) 294-306.

[5] B. Degrenne, J. Pruvost, J. Legrand, Effect of prolonged hypoxia in autotrophic conditions in the hydrogen production by the green microalga Chlamydomonas reinhardtii in photobioreactor, Bioresour. Technol. 102 (2011) 1035-1043.

[6] O. Hu, M. Sommerfeld, E. Jarvis, M.L. Ghirardi, M. Posewitz, M. Seibert, A. Darzins, Microalgal triacylglycerols as feedstocks for biofuel production: perspectives and advances, Plant J. 54 (2008) 621-639.

[7] L. Rodolfi, G. Chini Zittelli, N. Bassi, G. Padovani, N. Biondi, G. Bonini, M. Tredici, Microalgae for oil: Strain selection, induction of lipid synthesis and outdoor mass cultivation in a low-cost photobioreactor, Biotechnol. Bioeng. 102 (2009) 100-112.

[8] A.P. Carvalho, L.A. Meireles, F.X. Malcata, Microalgal reactors: a review of enclosed system designs and performances, Biotechnol. Prog. 22 (2006) 1490-1506.

[9] F. Lehr, C. Posten, Closed photo-bioreactors as tools for biofuel production, Curr. Opin. Biotechnol. 20 (2009) 280-285.

[10] C.U. Ugwu, H. Aoyagia, H. Uchiyamaa, Photobioreactors for mass cultivation of algae, Bioresour. Technol. 99 (2008) 4021-4028.

[11] J. Pruvost, Cultivation of algae in photobioreactors for biodiesel production, in: E.I. USA (Ed.), Biofuels: Alternative Feedstocks and Conversion Processes, A Pandey, C Larroche, SC Ricke and CG Dussap 2011, pp. 439-464.

[12] M. Morweiser, O. Kruse, B. Hankamer, C. Posten, Developments and perspectives of photobioreactors for biofuel production, Appl. Microbiol. Biotechnol. 87 (2010) 1291-1301.

[13] P.M. Slegers, M.B. Lösing, R.H. Wijffels, G. van Straten, A.J.B. van Boxtel, Scenario evaluation of open pond microalgae production, Algal Res. 2 (2013) 358-368.

[14] P.M. Slegers, P.J.M. van Beveren, R.H. Wijffels, G. van Straten, A.J.B. van Boxtel, Scenario analysis of large scale algae production in tubular photobioreactors, Appl. Energy 105 (2013) 395-406.

[15] P.M. Slegers, R.H. Wijffels, G. Van Straten, A.J.B. Van Boxtel, Design scenarios for flat panel photobioreactors, J. Appl. Energy 88 (2011) 3342-3353.

[16] J. Quinn, L. De Winter, T. Bradley, Microalgae bulk growth model with application to industrial scale systems, Bioresour. Technol. 102 (2011) 5083-5092.

[17] J. Pruvost, J.F. Cornet, V. Goetz, J. Legrand, Modeling dynamic functioning of rectangular photobioreactors in solar conditions, AIChE J. 57 (2011) 1947-1960.
[18] J. Pruvost, J.F. Cornet, V. Goetz, J. Legrand, Theoretical investigation of biomass productivities achievable in solar rectangular photobioreactors for the cyanobacterium Arthrospira platensis, Biotechnol. Prog. 28 (2012) 699-714.

[19] J. Pruvost, J.F. Cornet, Knowledge models for engineering and optimization of photobioreactors, in: C.P.a. C.Walter (Ed.), Microalgal Biotechnology, De Gruyter GmbH \& Co. KG 2012, pp. 181-224.

[20] L. Gonzalez de la Vara, C. Gomez-Lojero, Participation of plastoquinone, cytochrome c553 and ferredoxin-NADP + oxido reductase in both photosynthesis and respiration in Spirulina maxima, Photosynth. Res. 8 (1986) 65-78.

[21] H. Takache, J.F. Cornet, J. Pruvost, Kinetic modeling of the photosynthetic growth of Chlamydomonas reinhardtii in a photobioreactor, Biotechnol. Prog. 28 (2012) 681-692.

[22] H. Takache, G. Christophe, J.F. Cornet, J. Pruvost, Experimental and theoretical assessment of maximum productivities for the microalgae Chlamydomonas reinhardtii in two different geometries of photobioreactors, Biotechnol. Prog. 26 (2010) 431-440.

[23] J.F. Cornet, C.G. Dussap, P. Cluzel, G. Dubertret, A structured model for simulation of cultures of the cyanobacterium Spirulina platensis in photobioreactors. 1. Coupling between light transfer and growth kinetics, Biotechnol. Bioeng. 40 (1992) 817-825.

[24] J.F. Cornet, C.G. Dussap, J.B. Gros, Conversion of radiant light energy in photobioreactors, AIChE J. 40 (1994) 1055-1066.

[25] J.C. Ogbonna, H. Tanaka, Night biomass loss and changes in biochemical composition of cells during light/dark cyclic culture of Chlorella pyrenoidosa, J. Ferment. Bioeng. 82 (1996) 558-564

[26] F. Le Borgne, J. Pruvost, Investigation and modeling of biomass decay rate in the dark and its potential influence on net productivity of solar photobioreactors for microalga Chlamydomonas reinhardtii and cyanobacterium Arthrospira platensis, Bioresour. Technol. 138 (2013) 271-276.

[27] J.F. Cornet, Etude cinétique et énergétique d'un photobioréacteur. Etablissement d'un modèle structuré. Applications à un écosystème clos artificiel, Université Paris XI Orsay, 1992.

[28] F. Le Borgne, Développement d'un photobioréacteur solaire intensifié en vue de la production à grande échelle de biomasse microalgale, Université de Nantes, SaintNazaire, 2011.

[29] E.G. Josrgensen, The adaptation of plankton algae IV. Light adaptation in different algal species, Physiol. Plant. 22 (1969) 1307-1315.

[30] R. Kandilian, E. Lee, L. Pilon, Radiation and optical properties of Nannochloropsis oculata grown under different irradiances and spectra, Bioresour. Technol. 137 (2013) 63-73

[31] L. Pottier, Modélisation de l'effet de l'hydrodynamique sur l'accès à la lumière: application à la croissance photosynthétique dans un réacteur de géométrie torique( $\mathrm{PhD}$ Thesis) Nantes University, France, 2005.

[32] J.F. Cornet, Calculation of optimal design and ideal productivities of volumetricallylightened photobioreactors using the constructal approach, Chem. Eng. Sci. 65 (2010) 985-998.

[33] E. Lee, J. Pruvost, X. He, R. Munipalli, L. Pilon, Design tool and guidelines for outdoor photobioreactors, Chem. Eng. Sci. 106 (2014) 18-29.

[34] H. Takache, G. Christophe, J.F. Cornet, J. Pruvost, Experimental and theoretical assessment of maximum productivities for the micro-algae Chlamydomonas reinhardtii in two different geometries of photobioreactors, Biotechnol. Prog. 26 (2010) 431-440.

[35] J.F. Cornet, C.G. Dussap, A simple and reliable formula for assessment of maximum volumetric productivities in photobioreactors, Biotechnol. Prog. 25 (2009) 424-435.

[36] R. Aris, Elementary Chemical Reactor Analysis, Butterworth-Heinemann, Dover, New York, 1999.

[37] P.G. Falkowski, J. LaRoche, Minireview: acclimation to spectral irradiance in algae, J. Phycol. 27 (1991) 8-14.

[38] J.U. Grobbelaar, L. Nebdal, V. Tichy, Influence of high light/dark fluctuations on photosynthetic characteristics of microalgae photoacclimated to different light intensities and implications for mass algal cultivation, J. Appl. Phycol. 8 (1996) 335-343.

[39] C. Zonneveld, Photoinhibition as affected by photoacclimation in phytoplankton: a model approach, J. Theor. Biol. 193 (1998) 115-123.

[40] M. Janssen, J. Tramper, L.R. Mur, R.H. Wijffels, Enclosed outdoor photobioreactors: light regime, photosynthetic efficiency, scale-up, and future prospects, Biotechnol. Bioeng. 81 (2003) 193-210.

[41] M.R. Tredici, G. Chini Zittelli, Efficiency of sunlight utilization: tubular versus flat photobioreactors, Biotechnol. Bioeng. 57 (1998) 187-197.

[42] X. Wu, J.C. Merchuk, A model integrating fluid dynamics in photosynthesis and photoinhibition processes, Chem. Eng. Sci. 56 (2001) 3527-3538.

[43] A.P. Carvalho, S.O. Silva, J.M. Baptista, F.X. Malcata, Light requirements in microalgal photobioreactors: an overview of biophotonic aspects, Appl. Microbiol. Biotechnol. 89 (2011) 1275-1288.

[44] S. Hindersin, M. Leupold, M. Kerner, D. Hanelt, Irradiance optimization of outdoor microalgal cultures using solar tracked photobioreactors, Bioprocess Biosyst. Eng. 36 (2013) 345-355.

[45] G. Torzillo, P. Accolla, E. Pinzani, J. Masojidek, In situ monitoring of chlorophyll fluorescence to assess the synergistic effect of low temperature and high irradiance stresses in Spirulina cultures grown outdoors in photobioreactors, J. Appl. Phycol. 8 (1996).

[46] L. Pottier, J. Pruvost, J. Deremetz, J.F. Cornet, J. Legrand, C.G. Dussap, A fully predictive model for one-dimensional light attenuation by Chlamydomonas reinhardtii in a torus reactor, Biotechnol. Bioeng. 91 (2005) 569-582.

[47] J.F. Cornet, L. Favier, C.G. Dussap, Modeling stability of photoheterotrophic continuous cultures in photobioreactors, Biotechnol. Prog. 19 (2003) 1216-1227.

[48] J. Pruvost, J.F. Cornet, J. Legrand, Hydrodynamics influence on light conversion in photobioreactors: an energetically consistent analysis, Chem. Eng. Sci. 63 (2008) 3679-3694 